\title{
Inexpensive guaranteed and efficient upper bounds on the algebraic error in finite element discretizations*
}

\author{
Jan Papež ${ }^{1}$ and Martin Vohralík ${ }^{2}$ \\ ${ }^{1}$ Institute of Mathematics of the Czech Academy of Sciences, Žitná 25, 11567, Prague, Czech \\ Republic. jan@papez.org \\ ${ }^{2}$ Inria Paris, 2 rue Simone Iff, 75589 Paris, France. \& Université Paris-Est, CERMICS \\ (ENPC), 77455 Marne-la-Vallée, France. martin.vohralik@inria.fr
}

February 18, 2021

\begin{abstract}
We present new constructions of (approximate) $\mathbf{H}(\operatorname{div}, \Omega)$-liftings of the algebraic residual leading to estimators of the algebraic error in $h$ and $p$ finite element discretizations of a model diffusion problem. The estimators provide guaranteed bounds without any uncomputable constants and they are globally efficient, similarly to some recent developments, but the cost of their construction is significantly reduced. We provide a set of numerical experiments to assess the performance of the new estimators.
\end{abstract}

\section{Introduction}

Numerical discretizations of partial differential equations typically give rise to large sparse systems of linear algebraic equations, which are often solved by iterative solvers. Then two natural questions arise: 1) what is the algebraic error on iteration $i$ ?; 2) when to stop the iterations of the algebraic solver?

Pioneering results on estimating and balancing the algebraic and discretization error components were proposed, e.g, by Brandt [12], Bank and Sherman [6], Bai and Brandt [5], Bank and Smith [7], Rüde [30, 31, 32], Oswald [27], Becker et al. [8], or more recently by, e.g., Arioli et al. [2, 1], Janssen and Kanschat [21], or Meidner et al. [24]. However, the available estimates often involve some unknown generic constants, which can limit their use in practice. Using so-called flux reconstruction techniques, a posteriori error estimates including algebraic error and avoiding uncomputable constants were derived, e.g., in Jiránek et al. [22], Ern and Vohralík [15], or Papež et al. [29], see also the references therein. However, these results still come with some limitations; namely additional iteration steps are required. This drawback was overcome by the estimates of [28] relying on a lifting of the algebraic residual that is carried out over a hierarchy of meshes with local, mutually independent discrete $\mathbf{H}(\operatorname{div}, \Omega)$ contributions. Here, a parallel with multigrid and domain decomposition techniques for $\mathbf{H}(\operatorname{div}, \Omega)$ relying on local problems, proposed in the work of Arnold et al. [3, 4], can be seen, where in [3] the authors construct a spectrally equivalent preconditioner for the operator I - grad div and in [4] multigrid solvers. From [28],

\footnotetext{
*This project has received funding from the European Research Council (ERC) under the European Union's Horizon 2020 research and innovation program (grant agreement No 647134 GATIPOR). The work of J. Papež was supported by the Grant Agency of the Czech Republic under grant no. 20-01074S in the framework of RVO 67985840.
} 
guaranteed, fully computable, and efficient bounds on the algebraic, discretization, and total errors in conforming $h$ and $p$ finite element discretizations become available.

In this contribution, we elaborate on [28] and present new constructions of discrete $\mathbf{H}(\operatorname{div}, \Omega)$-liftings of the algebraic residual leading to a posteriori estimators of the algebraic error in $h$ and $p$ finite element discretizations of a model diffusion problem. In comparison with the construction of the lifting proposed in [28], the cost of the new construction is significantly reduced, while all the theoretical properties are maintained; the estimators namely provide guaranteed bounds without any uncomputable constants and are globally efficient.

We note that the present bounds on the algebraic error in principle differ from the bounds derived using properties of a particular algebraic solver, such as in $[35,17,25]$ for the conjugate gradient method; see also the references therein. Our bounds are valid for an arbitrary solver. They can also be evaluated locally to estimate local distribution of the algebraic error, and can be naturally evaluated as one ingredient when estimating discretization and total errors. The drawback of our estimates, in comparison with the estimates in [35, 17, 25], though they are much cheaper than those in [28], is that they still induce a higher computational cost. To further reduce this cost, we also present an approximate lifting of the algebraic residual. This lifting can be seen as only working with lowest-degree piecewise polynomial Raviart-Thomas-Nédélec (RTN) spaces for any order $p$ of discretization, but we promote in Algorithm 4 below a construction that actually does not request any computer implementation of the RTN spaces, neither any solution of local problems in the RTN spaces. This lifting still provides a guaranteed and efficient upper bound on the algebraic error, whose quality, however, numerically slightly deteriorates with increasing the polynomial degree $p$ of the approximation.

The rest of this contribution is organized as follows. We start in Sec. 2 with a warning example where some commonly used estimators on the algebraic error critically fail. In Sec. 3, we introduce the hierarchy of meshes and piecewise polynomial spaces that are used in the constructions of our liftings and estimators. A cornerstone of our approach, a lowest-degree coarse grid solver, is introduced in Sec. 4. Then we recall the construction of the lifting from [28] in Sec. 5 and present two new constructions in Secs. 6 and 7. In Sec. 8, we then construct the approximate lifting in the lowest-degree polynomial space. In Sec. 9 we compare the sizes of the local problems solved within the constructions of Secs. 5-8 and comment on their computational complexity. The upper bounds on the algebraic error in the model diffusion problem are presented in Sec. 10, and their efficiency is proved in Sec. 11. Numerical performance of the estimates is then examined in Sec. 12. The paper ends with a concluding discussion in Sec. 13.

\section{Warning example}

The purpose of this section is to present a simple example in which commonly used estimators of the algebraic error based on the algebraic residual vector fail importantly. In contrast, the rigorously justified upper bounds on the algebraic error developed in this manuscript behave without flaw.

Consider the Poisson model problem of finding $u: \Omega \rightarrow \mathbb{R}$ such that

$$
-\Delta u=f \quad \text { in } \Omega, \quad u=0 \quad \text { on } \partial \Omega .
$$

We take here the domains $\Omega=(0,1)^{d}, d=1,2,3$, and choose the source term $f$ such that the exact solutions are respectively given by $u_{d=1}(x)=x(1-x), u_{d=2}(x, y)=x(1-x) y(1-y)$, and $u_{d=3}(x, y, z)=$ $x(1-x) y(1-y) z(1-z)$. Consider a simplicial mesh with maximal element diameter $H$ : uniform for $d=1$, composed of congruent isosceles triangles for $d=2$, and composed of tetrahedra generated using the FreeFEM $[19,20]$ command cube for $d=3$; the latter relies on the TetGen mesh generator [34]. Importantly, in one or two space dimensions, we also consider a regular refinement with mesh size $h=H / 2$, where each interval or triangle is cut into respectively 2 or 4 congruent intervals or triangles. 
In three space dimensions, a new mesh with desired mesh size $h=H / 2$ is generated. We denote respectively by $u_{H}^{\mathrm{ex}}$ and $u_{h}^{\mathrm{ex}}$ the associated Galerkin finite element solutions, obtained with continuous and piecewise affine polynomials with respect to the meshes $\mathscr{T}_{H}$ and $\mathscr{T}_{h}$ and an exact algebraic solve. The associated coefficients in the standard nodal Lagrange basis on the mesh $\mathscr{T}_{h}$ are respectively denoted by $\mathrm{U}_{h}$ (we do not use the notation $\mathrm{U}_{H}$ since $u_{H}^{\mathrm{ex}}$ is henceforth percept on the mesh $\mathscr{T}_{h}$ ) and $\mathrm{U}_{h}^{\text {ex }}$, and the stiffness matrix on the mesh $\mathscr{T}_{h}$ is named $\mathbb{A}_{h}$, so that $\mathbb{A}_{h} \mathrm{U}_{h}^{\text {ex }}=\mathrm{F}_{h}$ but $\mathbb{A}_{h} \mathrm{U}_{h} \neq \mathrm{F}_{h}$.

We elaborate on [9, Example 4.6], where the one-dimensional case is presented with a thorough discussion. The point is to view $u_{H}^{\mathrm{ex}}$ as an approximate algebraic solution to $u_{h}^{\mathrm{ex}}$. There is no (iterative) algebraic solver here; we focus on estimating the energy norm of the algebraic error $\left\|\nabla\left(u_{h}^{\mathrm{ex}}-u_{H}^{\mathrm{ex}}\right)\right\|$, or, equivalently $\left\|\mathrm{U}_{h}^{\text {ex }}-\mathrm{U}_{h}\right\|_{\mathbb{A}_{h}}$ in one and two space dimensions, when varying the mesh size $h$. We consider the probably most widespread algebraic error estimate given by the Euclidean norm $\left\|\mathrm{R}_{h}\right\|_{2}$ of the algebraic residual vector $\mathrm{R}_{h}$ associated with $u_{H}^{\mathrm{ex}}, \mathrm{R}_{h}:=\mathrm{F}_{h}-\mathbb{A}_{h} \mathrm{U}_{h}$. It is known that $\left\|\mathrm{R}_{h}\right\|_{2}$ can be largely disconnected from the error. For this reason, we also plot the value $\left\|\mathbb{A}_{h}^{-1}\right\|_{2}^{1 / 2}\left\|R_{h}\right\|_{2}$, which provides a (worst-case) guaranteed upper bound on the algebraic error $\left\|\nabla\left(u_{h}^{\mathrm{ex}}-u_{H}^{\mathrm{ex}}\right)\right\|$ (see, e.g., the discussion in [29, Sec. 3.1]). The results are given in Fig. 1.
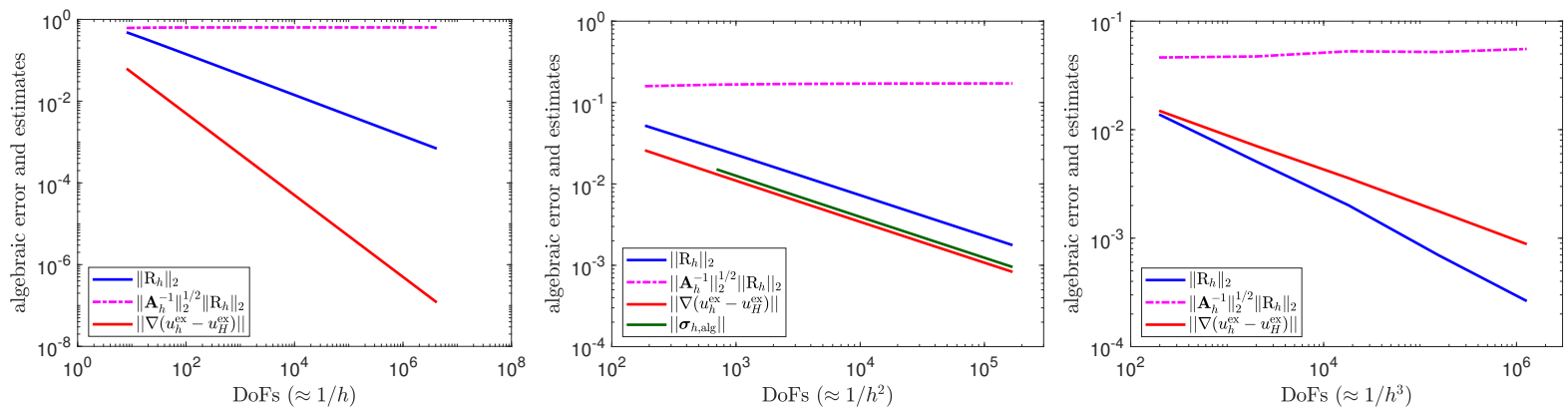

Figure 1: Unit $d$-cube problem with $u_{H}^{\mathrm{ex}}$ as an approximate algebraic solution to $u_{h}^{\mathrm{ex}}$. The algebraic error $\left\|\nabla\left(u_{h}^{\mathrm{ex}}-u_{H}^{\mathrm{ex}}\right)\right\|$, the norm $\left\|\mathrm{R}_{h}\right\|_{2}$ of the associated algebraic residual vector, and the guaranteed upper bound $\left\|\mathbb{A}_{h}^{-1}\right\|_{2}^{1 / 2}\left\|\mathrm{R}_{h}\right\|_{2}$ for the varying mesh size $h$ in one (left), two (middle), and three space dimensions (right). For $d=2$, we also plot the guaranteed upper bound $\eta_{6.3}^{p}=\left\|\sigma_{h, \text { alg }}\right\|$ on the algebraic error from (10.9b)

We can see that the Euclidean norm of the algebraic residual vector $\left\|\mathrm{R}_{h}\right\|_{2}$ behaves with decreasing $h$ differently for each space dimension. Though in two space dimensions, it happens to have the correct convergence rate and to be rather sharp, it dangerously over(under)estimates the algebraic error in one (three) space dimensions. The upper bound $\left\|\mathbb{A}_{h}^{-1}\right\|_{2}^{1 / 2}\left\|\mathrm{R}_{h}\right\|_{2}$ provides in all dimensions guaranteed, but misleading, information on the energy norm of the error, further deteriorating with mesh refinement. Finally, in the two-dimensional case, the guaranteed upper bound $\eta_{6.3}^{p}=\left\|\sigma_{h, \text { alg }}\right\|$ on the algebraic error, proposed in Sec. 6 of this work, is depicted to show that it tightly follows the algebraic error, in adequacy with the theory developed.

\section{Setting}

This section introduces the piecewise polynomial spaces used in the construction of our liftings and estimators.

\subsection{Hierarchy of meshes}

Let $\Omega \subset \mathbb{R}^{d}, 1 \leq d \leq 3$, be an open bounded polytope with a Lipschitz-continuous boundary. Let $\mathscr{T}_{h}$ be a simplicial mesh of $\Omega$, matching in the sense that for two distinct elements $K$ of $\mathscr{T}_{h}$, their intersection 
is either an empty set or a common vertex, edge, or face. Associated with $\mathscr{T}_{h}$, let there be a hierarchy of meshes $\left\{\mathscr{T}_{j}\right\}_{0 \leq j \leq J}$. These are again matching simplicial partitions of the domain $\Omega$, nested in the sense that $\mathscr{T}_{j}$ is a refinement of $\mathscr{T}_{j-1}, 1 \leq j \leq J$, and satisfying $\mathscr{T}_{h}=\mathscr{T}_{J}$. The set of vertices of $\mathscr{T}_{j}$ is denoted by $\mathscr{V}_{j}$, and it is decomposed into interior vertices $\mathscr{V}_{j}^{\text {int }}$ and boundary vertices $\mathscr{V}_{j}^{\text {ext }}$. By $\psi_{j}^{\mathbf{a}}$, we denote the standard hat function associated with the vertex $\mathbf{a} \in \mathscr{V}_{j}, 0 \leq j \leq J$, i.e., the function that is piecewise affine with respect to the $j$-th level mesh $\mathscr{T}_{j}$, taking the value 1 in vertex a and zero in all other $j$-th level vertices of $\mathscr{V}_{j}$. The interior of the support of $\psi_{j}^{\mathrm{a}}$ is denoted by $\omega_{j}^{\mathrm{a}}$ and it corresponds to the patch of elements of $\mathscr{T}_{j}$ which share the vertex $\mathbf{a} \in \mathscr{V}_{j}$. For $\mathbf{a} \in \mathscr{V}_{j-1}, 1 \leq j \leq J$, we denote by $\mathscr{V}_{j}^{\mathbf{a}}$, the subset of vertices of the finer level $\mathscr{V}_{j}$ that lie in the closure of the patch subdomain $\overline{\omega_{j-1}^{\mathrm{a}}}$. Finally, we identify $\omega_{h}^{\mathbf{a}}:=\omega_{J}^{\mathrm{a}}$ and $\mathscr{V}_{h}:=\mathscr{V}_{J}, \mathscr{V}_{h}^{\text {int }}:=\mathscr{V}_{J}^{\text {int }}, \mathscr{V}_{h}^{\text {ext }}:=\mathscr{V}_{J}^{\text {ext }}$ on the computational mesh that corresponds to the finest level $J$.

\subsection{Finite element approximation spaces}

The $p$ th-order conforming finite element space associated with the mesh $\mathscr{T}_{j}$ is denoted by $V_{j}^{p}$ and defined as

$$
V_{j}^{p}:=\mathbb{P}^{p}\left(\mathscr{T}_{j}\right) \cap H_{0}^{1}(\Omega), \quad 1 \leq p, 0 \leq j \leq J,
$$

where piecewise polynomials of total degree at most $q$ on each element are given by

$$
\mathbb{P}^{q}\left(\mathscr{T}_{j}\right):=\left\{v_{j} \in L^{2}(\Omega),\left.v_{j}\right|_{K} \in \mathbb{P}^{q}(K) \quad \forall K \in \mathscr{T}_{j}\right\}, \quad 0 \leq q, 0 \leq j \leq J .
$$

In the following, the polynomial degree $p$ will be fixed; we will use the notation $V_{h}^{p}:=V_{J}^{p}$. By $(\cdot, \cdot)$ we denote the $L^{2}(\Omega)$ or $\left[L^{2}(\Omega)\right]^{d}$ scalar product and by $\Pi_{j}^{q}$ the $L^{2}(\Omega)$-orthogonal projection onto $\mathbb{P}^{q}\left(\mathscr{T}_{j}\right)$.

\subsection{Finite element spaces for $\mathbf{H}(\operatorname{div}, \Omega)$-liftings}

For our estimates, we construct $\mathbf{H}(\operatorname{div}, \Omega)$-conforming vector-valued liftings in the RTN finite element spaces $\mathbf{V}_{j}^{q} \subset \mathbf{H}(\operatorname{div}, \Omega), q \geq 0$. These contain vector-valued piecewise polynomials with continuous normal trace and are given by

$$
\mathbf{V}_{j}^{q}:=\left\{\mathbf{v}_{j} \in \mathbf{H}(\operatorname{div}, \Omega) ;\left.\mathbf{v}_{j}\right|_{K} \in\left[\mathbb{P}^{q}(K)\right]^{d}+\mathbb{P}^{q}(K) \mathbf{x} \quad \forall K \in \mathscr{T}_{j}\right\},
$$

see, e.g., Boffi et al. [10]. In particular, we consider either $q=0$ or $q=p$ where, recall, $p$ is the polynomial degree of the finite element approximation space $V_{h}^{p}$.

The constructions below use also the local subspaces

$$
\begin{array}{rlrl}
\mathbf{V}_{j}^{q}\left(\omega_{j-1}^{\mathbf{a}}\right) & :=\left\{\left.\mathbf{v}_{j} \in \mathbf{V}_{j}^{q}\right|_{\omega_{j-1}^{\mathrm{a}}} ; \mathbf{v}_{j} \cdot \mathbf{n}_{\omega_{j-1}^{\mathrm{a}}}=0 \text { on } \partial \omega_{j-1}^{\mathrm{a}}\right\}, & & \\
Q_{j}^{q}\left(\omega_{j-1}^{\mathbf{a}}\right) & :=\left\{\left.q_{j} \in \mathbb{P}^{q}\left(\mathscr{T}_{j}\right)\right|_{\omega_{j-1}^{\mathrm{a}}} ^{\mathrm{a}} ;\left(q_{j}, 1\right) \omega_{j-1}^{\mathrm{ant}}=0\right\}, & & \\
\mathbf{V}_{j}^{q}\left(\omega_{j-1}^{\mathbf{a}}\right) & :=\left\{\left.\mathbf{v}_{j} \in \mathbf{V}_{j}^{q}\right|_{\omega_{j-1}^{\mathrm{a}}} ; \mathbf{v}_{j} \cdot \mathbf{n}_{\omega_{j-1}^{\mathrm{a}}}=0 \text { on } \partial \omega_{j-1}^{\mathbf{a}} \backslash \partial \Omega\right\}, & & \mathbf{a} \in \mathscr{V}_{j-1}^{\text {ext }} \\
Q_{j}^{q}\left(\omega_{j-1}^{\mathbf{a}}\right) & :=\left\{\left.q_{j} \in \mathbb{P}^{q}\left(\mathscr{T}_{j}\right)\right|_{\omega_{j-1}^{\mathrm{a}}}\right\}, &
\end{array}
$$

The degrees of freedom of the space $\mathbf{V}_{j}^{q}\left(\omega_{j-1}^{\mathbf{a}}\right)$ for $q=1$ are illustrated in Fig. 3 .

The constructions of the liftings in Sec. 6 and 7 use the lowest-degree functions with $q=0$ on the intermediate levels $1 \leq j<J$ and the highest-degree functions with $q=p$ on the finest level $j=J$, whereas the construction of Sec. 8 uses $q=0$ on all levels $1 \leq j \leq J$. The lowest-degree RTN functions with $q=0$ have one degree of freedom associated to each ( $(d-1)$-dimensional) face (vertex for $d=1$, edge for $d=2$, face for $d=3$ ), see Figure 2, left, for a single element when $d=2$. Moreover, the basis 
of $\mathbf{V}_{j}^{0}$ can be constructed using the geometry only: the basis function $\mathbf{v}_{F}$ associated with a face $F$ shared by two simplices $K_{+}$and $K_{-}$can be defined by

$$
\mathbf{v}_{F}(\mathbf{x}):= \begin{cases}\frac{|F|}{d\left|K_{+}\right|}\left(\mathbf{x}-\mathbf{x}_{F,+}\right) & \text { if } \mathbf{x} \in K_{+} \\ -\frac{|F|}{d\left|K_{-}\right|}\left(\mathbf{x}-\mathbf{x}_{F,-}\right) & \text { if } \mathbf{x} \in K_{-} \\ \mathbf{0} & \text { otherwise }\end{cases}
$$

where $|F|$ denotes the surface of $F,|K|$ denotes the area of $K$, and $\mathbf{x}_{F,+}, \mathbf{x}_{F,-}$ are the vertices opposite to $F$ in the elements $K_{+}$and $K_{-}$, respectively. For an illustration when $d=2$, see Figure 2, right.
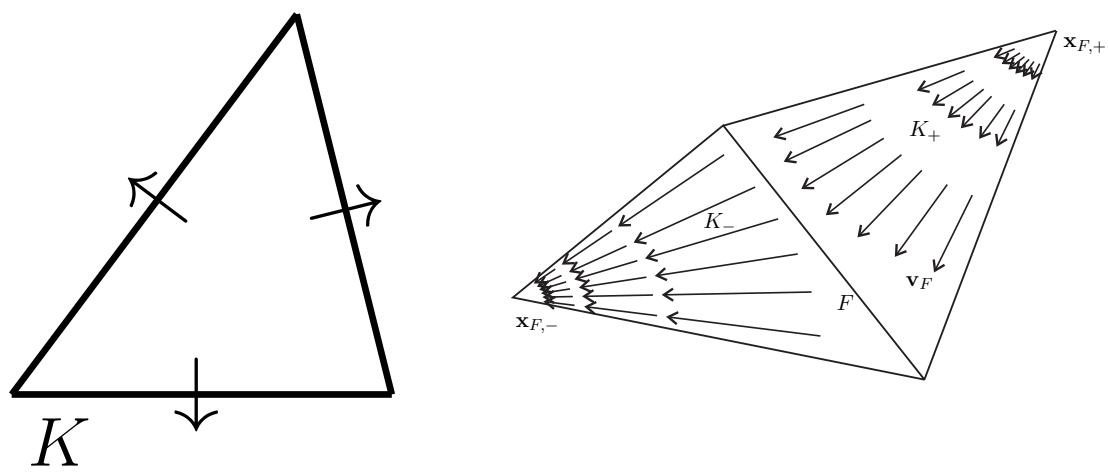

Figure 2: Lowest-degree RTN functions in two space dimensions: degrees of freedom on a single element $K$ (left) and a basis function associated with a face $F$ (right).

\subsection{Operations needed for the constructions}

In this section, we list operations with piecewise polynomial functions that are used in the forthcoming constructions.

On each level $1 \leq j \leq J$, we need elementwise products on each simplex $K \in \mathscr{T}_{j}$

- $\left(\mathbf{A} \nabla v_{h}, \nabla w_{h}\right)_{K},\left(\nabla v_{h}, \nabla w_{h}\right)_{K},\left(v_{h}, w_{h}\right)_{K}, \quad v_{h}, w_{h} \in \mathbb{P}^{q}(K)$,

- $\left(\mathbf{A}^{-1} \mathbf{v}_{j}, \mathbf{w}_{j}\right)_{K},\left(v_{h}, \nabla \cdot \mathbf{v}_{j}\right)_{K}, \quad \mathbf{v}_{j}, \mathbf{w}_{j} \in \mathbf{V}_{j}^{q}, v_{h} \in \mathbb{P}^{q}(K)$.

For $r_{h} \in \mathbb{P}^{q}\left(\mathscr{T}_{h}\right)$, a piecewise polynomial function defined on the finest mesh, we will evaluate on each level $0 \leq j \leq J$ and each element $K \in \mathscr{T}_{j}$

- $\left(r_{h}, v_{j}\right)_{K}, \quad v_{j} \in \mathbb{P}^{q}(K)$,

- $\left(\psi_{j-1} r_{h}, v_{j}\right)_{K}, \quad v_{j} \in \mathbb{P}^{q}(K), \psi_{j-1} \in \mathbb{P}^{1}(K)$.

On the first level we will need, for each $K \in \mathscr{T}_{1}$,

- $\left(\mathbf{A} \nabla \psi_{0} \cdot \nabla \phi_{0}, v_{1}\right)_{K}, \quad \psi_{0}, \phi_{0} \in \mathbb{P}^{1}(K), v_{1} \in \mathbb{P}^{q}(K)$,

and for each level $1 \leq j \leq J$ and each element $K \in \mathscr{T}_{j}$,

- $\left(\nabla \psi_{j} \cdot \nabla \phi_{j}, v_{j}\right)_{K}, \quad \psi_{j}, \phi_{j} \in \mathbb{P}^{1}(K), v_{j} \in \mathbb{P}^{q}(K)$.

On each level $1<j \leq J$, we will use the $L^{2}$-orthogonal projections $\Pi_{j}^{q}$ and $\Pi_{j-1}^{q}$.

Finally, we have to sum RTN functions from different levels, i.e., to evaluate, for each $1<j \leq J$,

- $\mathbf{v}_{j}+\mathbf{v}_{j-1}, \quad \mathbf{v}_{j} \in \mathbf{V}_{j}^{q}, \mathbf{v}_{j-1} \in \mathbf{V}_{j-1}^{q^{\prime}}$, with $0 \leq q^{\prime} \leq q$.

Note, however, that no operations with RTN functions are needed in the inexpensive construction of Sec. 8, detailed in Algorithm 4. 


\section{Coarsest-level Riesz representer}

Let $r_{h} \in \mathbb{P}^{p}\left(\mathscr{T}_{h}\right)$ and $\mathbf{A} \in\left[\mathbb{P}^{0}\left(\mathscr{T}_{h}\right)\right]^{d \times d}$. We will use below as a crucial step in the construction of the liftings a piecewise affine Riesz representer $\rho_{0, \text { alg }} \in V_{0}^{1}$ of $r_{h}$,

$$
\left(\mathbf{A} \nabla \rho_{0, \mathrm{alg}}, \nabla v_{0}\right)=\left(r_{h}, v_{0}\right) \quad \forall v_{0} \in V_{0}^{1} .
$$

This procedure is familiar from the coarsest-grid residual solve in multigrid methods, see, e.g., [18, Section 2.5]. The piecewise affine Riesz representer is also in the heart of estimating the algebraic error in [26].

The size of the algebraic system associated with (4.1) is equal to $\left|\mathscr{V}_{0}^{\text {int }}\right|$, the number of interior vertices of the coarsest mesh $\mathscr{T}_{0}$. The cost of solving such system using the Cholesky decomposition is approximately $\left(\left|\mathscr{V}_{0}^{\text {int }}\right|\right)^{3} / 3$.

\section{$5 \mathbf{H}(\operatorname{div}, \Omega)$-lifting of a given piecewise polynomial with large patch solves}

In [28, Def. 4.3], a $\mathbf{H}(\operatorname{div}, \Omega)$-lifting of the given piecewise polynomial $r_{h} \in \mathbb{P}^{p}\left(\mathscr{T}_{h}\right)$ was introduced such that

$$
\begin{array}{r}
\boldsymbol{\sigma}_{h, \text { alg }} \in \mathbf{V}_{J}^{p}, \nabla \cdot \boldsymbol{\sigma}_{h, \text { alg }}=r_{h}, \text { contributions to }\left\|\mathbf{A}^{-1 / 2} \boldsymbol{\sigma}_{h, \text { alg }}\right\| \text { are locally minimized } \\
\text { with degree } p \text { on large patches } \omega_{j-1}^{\mathbf{a}} \text { of Figure } 3 .
\end{array}
$$

This is the basis of our developments. We now recall it here:

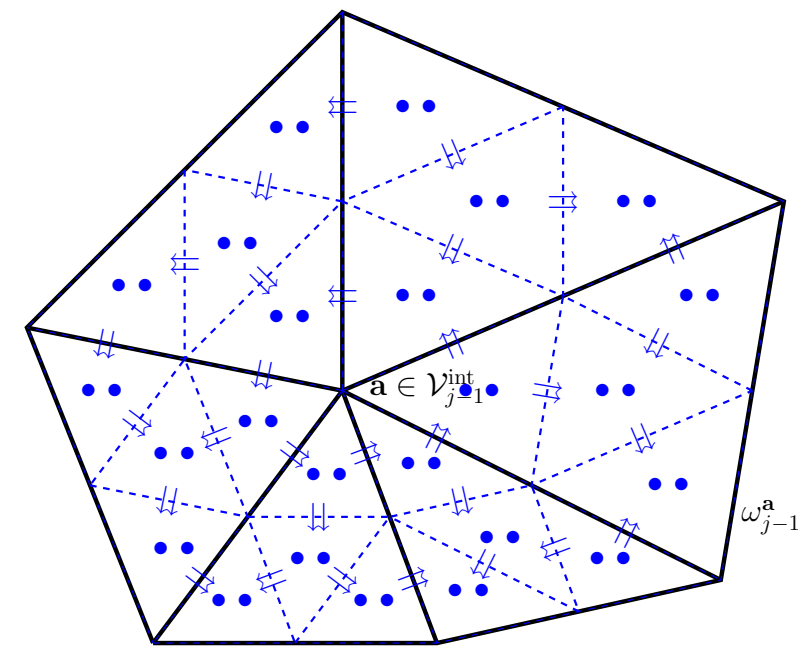

$$
\begin{gathered}
\text { coarse patch subdomain } \omega_{j-1}^{\mathrm{a}} \text { for } \mathbf{a} \in \mathscr{V}_{j-1}^{\text {int }} \\
\text { coarse mesh } \mathscr{T}_{j-1} \text { of } \omega_{j-1}^{\mathrm{a}} \text { (full line) } \\
\text { fine mesh } \mathscr{T}_{j} \text { of } \omega_{j-1}^{\mathbf{a}} \text { (dashed line) }
\end{gathered}
$$

degrees of freedom of the space $\mathbf{V}_{j}^{1}\left(\omega_{j-1}^{\mathbf{a}}\right)$ for the $\mathbf{H}($ div $)$ local Neumann solve on large patch subdomains (arrows and bullets)

Figure 3: $\mathbf{H}(\operatorname{div}, \Omega)$-lifting of Construction 5.1 by the large patch RTN solve; degrees of freedom of local RTN space $\mathbf{V}_{j}^{1}\left(\omega_{j-1}^{\mathbf{a}}\right)$ from (3.1)

Construction $5.1\left(\mathbf{H}(\operatorname{div}, \Omega)\right.$-lifting with large patch RTN solves). Given $r_{h} \in \mathbb{P}^{p}\left(\mathscr{T}_{h}\right)$, $\mathbf{A} \in\left[\mathbb{P}^{0}\left(\mathscr{T}_{h}\right)\right]^{d \times d}$, and the corresponding coarsest-level Riesz representer $\rho_{0, \mathrm{alg}} \in V_{0}^{1}$ given by (4.1), 
construct $\sigma_{h, \text { alg }} \in \mathbf{V}_{J}^{p}$ as a hierarchical sum

$$
\sigma_{h, \mathrm{alg}}:=\sum_{j=1}^{J} \sum_{\mathbf{a} \in \mathscr{Y}_{j-1}} \sigma_{j, \mathrm{alg}}^{\mathbf{a}},
$$

where the local contributions $\boldsymbol{\sigma}_{j, \text { alg }}^{\mathbf{a}} \in \mathbf{V}_{j}^{p}\left(\omega_{j-1}^{\mathbf{a}}\right)$ solve the mixed finite element problems: find $\left(\boldsymbol{\sigma}_{j, \text { alg }}^{\mathbf{a}}, \gamma_{j}^{\mathbf{a}}\right) \in$ $\mathbf{V}_{j}^{p}\left(\omega_{j-1}^{\mathbf{a}}\right) \times Q_{j}^{p}\left(\omega_{j-1}^{\mathbf{a}}\right)$ such that

$$
\begin{array}{rlrl}
\left(\mathbf{A}^{-1} \boldsymbol{\sigma}_{j, \text { alg }}^{\mathbf{a}}, \mathbf{v}_{j}\right)_{\omega_{j-1}^{\mathrm{a}}}-\left(\gamma_{j}^{\mathbf{a}}, \nabla \cdot \mathbf{v}_{j}\right)_{\omega_{j-1}^{\mathrm{a}}} & =0 & & \forall \mathbf{v}_{j} \in \mathbf{V}_{j}^{p}\left(\omega_{j-1}^{\mathbf{a}}\right), \\
\left(\nabla \cdot \boldsymbol{\sigma}_{j, \text { alg }}^{\mathbf{a}}, q_{j}\right)_{\omega_{j-1}^{\mathrm{a}}}=\left(g^{\mathbf{a}, j}, q_{j}\right)_{\omega_{j-1}^{\mathrm{a}}} & & \forall q_{j} \in Q_{j}^{p}\left(\omega_{j-1}^{\mathbf{a}}\right),
\end{array}
$$

with

$$
g^{\mathbf{a}, j}:= \begin{cases}r_{h} \psi_{0}^{\mathbf{a}}-\mathbf{A} \nabla \rho_{0, \text { alg }} \cdot \nabla \psi_{0}^{\mathbf{a}} & j=1, \\ \left(\mathrm{Id}-\Pi_{j-1}^{p}\right)\left(r_{h} \psi_{j-1}^{\mathbf{a}}\right) & 1<j \leq J .\end{cases}
$$

We note that the equivalent formulation of problem (5.3) is

$$
\boldsymbol{\sigma}_{j, \mathrm{alg}}^{\mathbf{a}}=\underset{\substack{\mathbf{v}_{j} \in \mathbf{V}_{j}^{p}\left(\omega_{j-1}^{\mathbf{a}}\right) \\ \nabla \cdot \mathbf{v}_{j}=\Pi_{j}^{p} \mathbf{a}^{2}, j}}{\arg \min }\left\|\mathbf{A}^{-1 / 2} \mathbf{v}_{j}\right\|_{\omega_{j-1}^{\mathrm{a}}} .
$$

Construction 5.1 is presented in an algorithmic form in Algorithm 1. Its drawback is the computational cost of the local solves in (5.3) (recall the degrees of freedom of $\mathbf{V}_{j}^{1}\left(\omega_{j-1}^{\mathbf{a}}\right)$ for $p=1$ in Fig. 3). Some possibilities of computational simplifications while not changing Construction 5.1 are presented in [28, Sec. 8], but all these possibilities still involve solving at least some problems of the form (5.3). In the following sections, we present three ways how to replace the local solve (5.3) or (5.5) by a much cheaper procedure, while still maintaining all important theoretical properties.

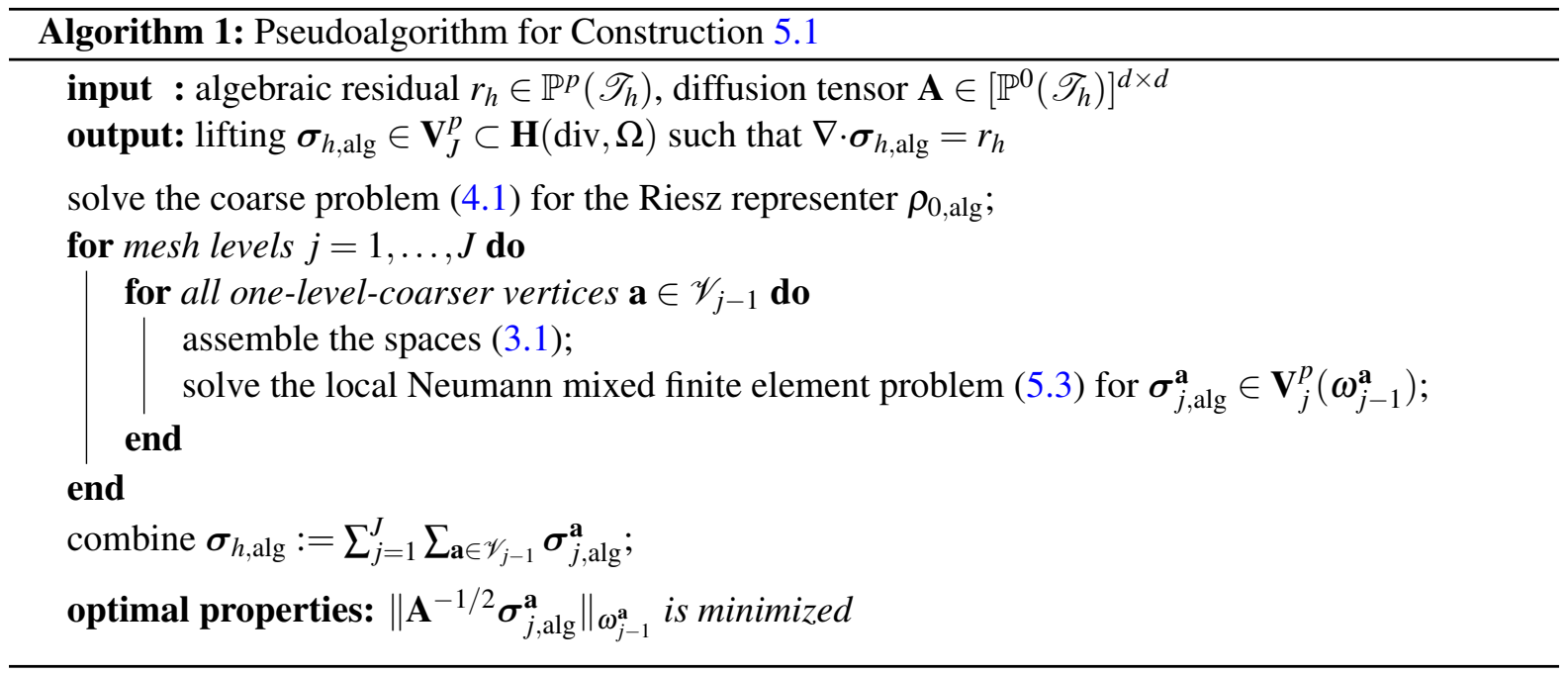

\section{$6 \mathrm{H}(\operatorname{div}, \Omega)$-lifting of a given piecewise polynomial with small patch solves}

The construction presented in this section produces

$$
\begin{gathered}
\boldsymbol{\sigma}_{h, \text { alg }} \in \mathbf{V}_{J}^{p}, \nabla \cdot \boldsymbol{\sigma}_{h, \text { alg }}=r_{h}, \text { contributions to }\left\|\mathbf{A}^{-1 / 2} \boldsymbol{\sigma}_{h, \text { alg }}\right\| \text { are locally minimized } \\
\text { with degree } p(j=J) \text { and with degree } 0(j<J) \\
\text { on small patches } \omega^{\mathbf{a}, \mathbf{a}^{\prime}} \text { of Figure } 4 .
\end{gathered}
$$


It ensures $\boldsymbol{\sigma}_{h, \text { alg }} \in \mathbf{V}_{J}^{p}$ and $\nabla \cdot \boldsymbol{\sigma}_{h, \text { alg }}=r_{h}$ as in (5.1), but differs from Construction 5.1 in two key features. First, the contributions on the intermediate levels $1 \leq j<J$ are computed in the lowest-degree RTN space $\mathbf{V}_{j}^{0}$, in place of the much larger $\mathbf{V}_{j}^{p}$. Second, the minimizations (5.3) on all levels $1 \leq j \leq J$ and over every patch subdomain $\omega_{j-1}^{\mathbf{a}}, \mathbf{a} \in \mathscr{V}_{j-1}$, are replaced by a single piecewise affine conforming FEM problem on the large patch subdomain $\omega_{j-1}^{\mathrm{a}}$ and several smaller problems posed on small patch subdomains $\omega^{\mathbf{a}, \mathbf{a}^{\prime}}, \mathbf{a}^{\prime} \in \mathscr{V}_{j}^{\mathbf{a}}$, where, recall, $\mathscr{V}_{j}^{\mathbf{a}}$ denotes the set of vertices of $\mathscr{V}_{j}$ that lie in $\overline{\omega_{j-1}^{\mathbf{a}}}$ and

$$
\omega^{\mathbf{a}, \mathbf{a}^{\prime}}:=\omega_{j}^{\mathbf{a}^{\prime}} \cap \omega_{j-1}^{\mathbf{a}}, \quad \mathbf{a}^{\prime} \in \mathscr{V}_{j}^{\mathbf{a}} ;
$$

see Figure 4. This significantly reduces the size of the associated local linear algebraic systems (cf. the detailed discussion on a concrete example in Sec. 9 below). Denote

$$
\begin{array}{ll}
H_{\star}^{1}\left(\omega_{j-1}^{\mathbf{a}}\right):=\left\{v \in H^{1}\left(\omega_{j-1}^{\mathbf{a}}\right),(v, 1)_{\omega_{j-1}^{\mathbf{a}}}=0\right\} & \mathbf{a} \in \mathscr{V}_{j-1}^{\mathrm{int}}, \\
H_{\star}^{1}\left(\omega_{j-1}^{\mathbf{a}}\right):=\left\{v \in H^{1}\left(\omega_{j-1}^{\mathbf{a}}\right), v=0 \text { on } \partial \Omega\right\} & \mathbf{a} \in \mathscr{V}_{j-1}^{\text {ext }},
\end{array}
$$

and (cf. (3.1)),

$$
\begin{array}{rlrl}
\mathbf{V}_{j}^{p^{\prime}}\left(\omega^{\mathbf{a}, \mathbf{a}^{\prime}}\right):=\left\{\left.\mathbf{v}_{j} \in \mathbf{V}_{j}^{p^{\prime}}\right|_{\omega^{\mathbf{a}, \mathbf{a}^{\prime}}} ; \mathbf{v}_{j} \cdot \mathbf{n}_{\omega^{\mathbf{a}, \mathbf{a}^{\prime}}}=0 \text { on } \partial \omega^{\mathbf{a}, \mathbf{a}^{\prime}} \backslash \partial \Omega\right\}, & \\
Q_{j}^{p^{\prime}}\left(\omega^{\mathbf{a}, \mathbf{a}^{\prime}}\right):=\left\{\left.q_{j} \in \mathbb{P}^{p^{\prime}}\left(\mathscr{T}_{j}\right)\right|_{\omega^{\mathbf{a}, \mathbf{a}^{\prime}}}\right\} & \\
\mathbf{V}_{j}^{p^{\prime}}\left(\omega^{\mathbf{a}, \mathbf{a}^{\prime}}\right):=\left\{\left.\mathbf{v}_{j-1}^{\text {ext }} \wedge \mathbf{V}_{j}^{p^{\prime}}\right|_{\omega^{\mathbf{a}, \mathbf{a}^{\prime}}} ; \mathbf{v}_{j} \in \mathscr{V}_{j}^{\text {ext }}, \mathbf{n}_{\omega^{\mathbf{a}, \mathbf{a}^{\prime}}}=0 \text { on } \partial \omega^{\mathbf{a}, \mathbf{a}^{\prime}}\right\}, & \\
Q_{j}^{p^{\prime}}\left(\omega^{\mathbf{a}, \mathbf{a}^{\prime}}\right):=\left\{\left.q_{j} \in \mathbb{P}^{p^{\prime}}\left(\mathscr{T}_{j}\right)\right|_{\omega^{\mathbf{a}, \mathbf{a}^{\prime}}} ;\left(q_{j}, 1\right)_{\omega^{\mathbf{a}, \mathbf{a}^{\prime}}}=0\right\}, & & \text { otherwise, }
\end{array}
$$

where the polynomial degree will be chosen as $p^{\prime}=0$ or $p^{\prime}=p$.

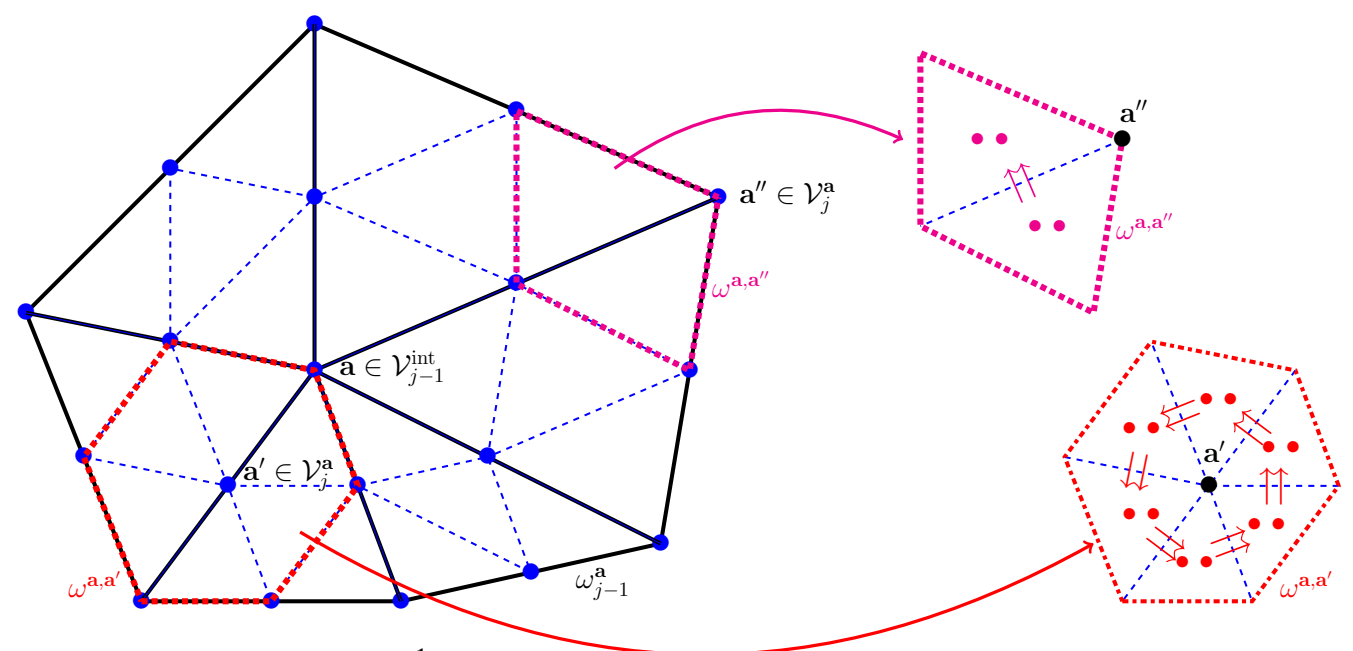

degrees of freedom for the $H^{1}$ Neumann solve on $\omega_{j-1}^{\mathbf{a}}$ (bullets), corresponding to the $j$-level vertices in $\mathscr{V}_{j}^{\mathbf{a}}$

degrees of freedom for the $\mathbf{H}($ div $)$ Neumann solve on the small patches $\omega^{\mathbf{a}, \mathbf{a}^{\prime}}$ ( $p^{\prime}=1$, arrows and bullets $)$

Figure 4: $\mathbf{H}(\operatorname{div}, \Omega)$-lifting of Construction 6.1 and Construction 6.3 by piecewise affine conforming FEM problem on the large patch subdomain $\omega_{j-1}^{\mathrm{a}}$ and smaller RTN problems on small patch subdomains $\omega^{\mathbf{a}, \mathbf{a}^{\prime}}, \mathbf{a}^{\prime} \in \mathscr{V}_{j}^{\mathbf{a}}$

Construction 6.1 (H(div, $\Omega)$-lifting eliminating large patch RTN solves). Given $r_{h} \in \mathbb{P}^{p}\left(\mathscr{T}_{h}\right), \mathbf{A} \in\left[\mathbb{P}^{0}\left(\mathscr{T}_{h}\right)\right]^{d \times d}$, and the corresponding coarsest-level Riesz representer $\rho_{0, \mathrm{alg}} \in V_{0}^{1}$ given by (4.1), set

$$
p^{\prime}:=0 \quad \text { for } 1 \leq j<J \quad \text { and } \quad p^{\prime}:=p \quad \text { for } j=J .
$$


Then, for each level $1 \leq j \leq J$

1. For each vertex $\mathbf{a} \in \mathscr{V}_{j-1}$, find $\left.\imath_{j}^{\mathbf{a}} \in \mathbb{P}^{1}\left(\mathscr{T}_{j}\right)\right|_{\omega_{j-1}^{\mathrm{a}}} \cap H_{\star}^{1}\left(\omega_{j-1}^{\mathrm{a}}\right)$, a continuous piecewise affine polynomial (with zero mean value on $\omega_{j-1}^{\mathbf{a}}$ if $\mathbf{a} \in \mathscr{V}_{j-1}^{\mathrm{int}}$ and vanishing on $\partial \Omega$ if $\mathbf{a} \in \mathscr{V}_{j-1}^{\text {ext }}$, see (6.2)), such that

$$
\left(\nabla l_{j}^{\mathbf{a}}, \nabla v_{j}\right)_{\omega_{j-1}^{\mathbf{a}}}=\left.\left(g^{I, \mathbf{a}, j}, v_{j}\right)_{\omega_{j-1}^{\mathbf{a}}} \quad \forall v_{j} \in \mathbb{P}^{1}\left(\mathscr{T}_{j}\right)\right|_{\omega_{j-1}^{\mathbf{a}}} \cap H_{\star}^{1}\left(\omega_{j-1}^{\mathbf{a}}\right),
$$

where

$$
g^{I, \mathbf{a}, j}:= \begin{cases}\Pi_{1}^{0}\left(r_{h} \psi_{0}^{\mathbf{a}}-\mathbf{A} \nabla \rho_{0, \mathrm{alg}} \cdot \nabla \psi_{0}^{\mathbf{a}}\right) & j=1, \\ \Pi_{j}^{p^{\prime}}\left(\mathrm{Id}-\Pi_{j-1}^{0}\right)\left(r_{h} \psi_{j-1}^{\mathbf{a}}\right) & 1<j \leq J .\end{cases}
$$

2. Run through all vertices $\mathbf{a}^{\prime} \in \mathscr{V}_{j}^{\mathbf{a}}$ (vertices included in $\overline{\omega_{j-1}^{\mathbf{a}}}$ ) and on each small patch subdo$\operatorname{main} \omega^{\mathbf{a}, \mathbf{a}^{\prime}}$, construct $\boldsymbol{\sigma}_{j, \mathrm{alg}}^{\mathbf{a}, \mathbf{a}^{\prime}} \in \mathbf{V}_{j}^{p^{\prime}}\left(\omega^{\mathbf{a}, \mathbf{a}^{\prime}}\right)$ such that

$$
\nabla \cdot \boldsymbol{\sigma}_{j, \text { alg }}^{\mathbf{a}, \mathbf{a}^{\prime}}=g^{I I, \mathbf{a}, \mathbf{a}^{\prime}, j},
$$

where

$$
g^{I I, \mathbf{a}, \mathbf{a}^{\prime}, j}:=\Pi_{j}^{p^{\prime}}\left(g^{I, \mathbf{a}, j} \psi_{j}^{\mathbf{a}^{\prime}}\right)-\nabla l_{j}^{\mathbf{a}} \cdot \nabla \psi_{j}^{\mathbf{a}^{\prime}} .
$$

Finally, define

$$
\boldsymbol{\sigma}_{j, \text { alg }}^{\mathbf{a}}:=\sum_{\mathbf{a}^{\prime} \in \mathscr{Y}_{j}^{\mathbf{a}}} \boldsymbol{\sigma}_{j, \mathrm{alg}}^{\mathbf{a}, \mathbf{a}^{\prime}}, \quad \sigma_{h, \text { alg }}:=\sum_{j=1}^{J} \sum_{\mathbf{a} \in \mathscr{Y}_{j-1}} \sigma_{j, \text { alg }}^{\mathbf{a}}
$$

Lemma 6.2 (Properties of $\sigma_{h, \text { alg }}$ ). Any lifting $\sigma_{h, \text { alg }}$ of Construction 6.1 satisfies

$$
\boldsymbol{\sigma}_{h, \mathrm{alg}} \in \mathbf{V}_{J}^{p}, \nabla \cdot \boldsymbol{\sigma}_{h, \mathrm{alg}}=r_{h}
$$

Proof. All the contributions $\boldsymbol{\sigma}_{j, \text { alg }}^{\mathbf{a}, \mathbf{a}^{\prime}}$ and $\boldsymbol{\sigma}_{j, \text { alg }}^{\mathbf{a}}$ extended by zero to $\Omega$ belong to $\mathbf{V}_{j}^{0}$, for $j<J$, and to $\mathbf{V}_{j}^{p}$, for $j=J$, so that $\sigma_{h, \text { alg }} \in \mathbf{V}_{J}^{p}$. For the divergence claim, let $\sigma_{j, \text { alg }}:=\sum_{\mathbf{a} \in \mathscr{H}_{j-1}} \boldsymbol{\sigma}_{j, \mathrm{alg}}^{\mathbf{a}}, 1 \leq j \leq J$. We rely on the two partitions of unity

$$
\sum_{\mathbf{a}^{\prime} \in \mathscr{Y}_{j}^{\mathbf{a}}} \psi_{j}^{\mathbf{a}^{\prime}}=1 \text { on } \omega_{j-1}^{\mathbf{a}} \quad \forall \mathbf{a} \in \mathscr{V}_{j-1}, 1 \leq j \leq J
$$

and

$$
\sum_{\mathbf{a} \in \mathscr{Y} \mathscr{V}_{j-1}} \psi_{j-1}^{\mathbf{a}}=1 \text { on } \Omega, \quad 1 \leq j \leq J
$$

and proceed in four steps.

Step 1: First, using the partition of unity (6.10) for any $1<j<J$, and owing to the choice (6.4), $\sigma_{j, \text { alg }}^{\mathrm{a}}$ satisfying (6.7)-(6.9) fulfills

$$
\nabla \cdot \boldsymbol{\sigma}_{j, \text { alg }}^{\mathbf{a}}=\sum_{\mathbf{a}^{\prime} \in \mathscr{Y}_{j}^{\mathbf{a}}} \nabla \cdot \boldsymbol{\sigma}_{j, \text { alg }}^{\mathbf{a}, \mathbf{a}^{\prime}}=\Pi_{j}^{0}\left[\Pi_{j}^{0}\left(\mathrm{Id}-\Pi_{j-1}^{0}\right)\left(r_{h} \psi_{j-1}^{\mathbf{a}}\right)\right]=\Pi_{j}^{0}\left(\mathrm{Id}-\Pi_{j-1}^{0}\right)\left(r_{h} \psi_{j-1}^{\mathbf{a}}\right) .
$$

Consequently, using the partition of unity (6.11),

$$
\nabla \cdot \boldsymbol{\sigma}_{j, \mathrm{alg}}=\sum_{\mathbf{a} \in \mathscr{Y}_{j-1}} \nabla \cdot \boldsymbol{\sigma}_{j, \mathrm{alg}}^{\mathbf{a}}=\Pi_{j}^{0}\left(\mathrm{Id}-\Pi_{j-1}^{0}\right) r_{h}=\Pi_{j}^{0} r_{h}-\Pi_{j-1}^{0} r_{h} .
$$


Step 2: On the first mesh $\mathscr{T}_{1}$, we similarly see that

$$
\nabla \cdot \boldsymbol{\sigma}_{1, \mathrm{alg}}^{\mathbf{a}}=\sum_{\mathbf{a}^{\prime} \in \mathscr{Y}_{1}^{\mathbf{a}}} \nabla \cdot \boldsymbol{\sigma}_{1, \mathrm{alg}}^{\mathbf{a} \mathbf{a}^{\prime}}=\Pi_{1}^{0}\left(r_{h} \psi_{0}^{\mathbf{a}}\right)-\mathbf{A} \nabla \rho_{0, \mathrm{alg}} \cdot \nabla \psi_{0}^{\mathbf{a}} \quad \text { and } \quad \nabla \cdot \boldsymbol{\sigma}_{1, \mathrm{alg}}=\Pi_{1}^{0} r_{h} .
$$

Step 3: On the finest mesh $\mathscr{T}_{J}$,

$$
\begin{aligned}
& \nabla \cdot \boldsymbol{\sigma}_{J, \text { alg }}^{\mathbf{a}}=\sum_{\mathbf{a}^{\prime} \in \mathscr{V}_{J}^{\mathbf{a}}} \nabla \cdot \boldsymbol{\sigma}_{J, \mathrm{alg}}^{\mathbf{a}, \mathbf{a}^{\prime}}=\Pi_{J}^{p}\left(\mathrm{Id}-\Pi_{J-1}^{0}\right)\left(r_{h} \psi_{J-1}^{\mathbf{a}}\right) \quad \text { and } \\
& \nabla \cdot \boldsymbol{\sigma}_{J, \mathrm{alg}}=\Pi_{J}^{p} r_{h}-\Pi_{J-1}^{0} r_{h}=r_{h}-\Pi_{J-1}^{0} r_{h} .
\end{aligned}
$$

Step 4: From (6.9) and the three above steps, we now conclude

$$
\nabla \cdot \boldsymbol{\sigma}_{h, \mathrm{alg}}=\sum_{j=1}^{J} \nabla \cdot \boldsymbol{\sigma}_{j, \mathrm{alg}}=\Pi_{1}^{0} r_{h}+\sum_{j=2}^{J-1}\left(\Pi_{j}^{0} r_{h}-\Pi_{j-1}^{0} r_{h}\right)+r_{h}-\Pi_{J-1}^{0} r_{h}=r_{h} .
$$

It remains to detail the construction of $\boldsymbol{\sigma}_{j, \mathrm{alg}}^{\mathbf{a}, \mathbf{a}^{\prime}} \in \mathbf{V}_{j}^{p^{\prime}}\left(\omega^{\mathbf{a}, \mathbf{a}^{\prime}}\right)$ in (6.7). The first construction is similar to Construction 5.1, but the local minimization is done over each small patch $\omega^{\mathbf{a}, \mathbf{a}^{\prime}}$, cf. Figure 4 , and it is therefore significantly cheaper, compare Figs 3 and 4.

Construction 6.3 (Local lifting $\boldsymbol{\sigma}_{j, \text { alg }}^{\mathbf{a} \mathbf{a}^{\prime}}$ by a small patch RTN solve). Let $1 \leq j \leq J$,

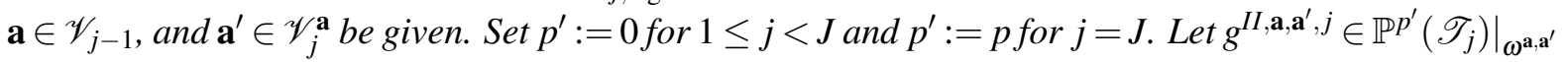
be given by (6.8). Define $\boldsymbol{\sigma}_{j, \text { alg }}^{\mathbf{a}, \mathbf{a}^{\prime}} \in \mathbf{V}_{j}^{p^{\prime}}\left(\omega^{\mathbf{a}, \mathbf{a}^{\prime}}\right)$ as the solution of the mixed finite element problem: find $\left(\boldsymbol{\sigma}_{j, \text { alg }}^{\mathbf{a}, \mathbf{a}^{\prime}}, \gamma_{j}^{\mathbf{a}, \mathbf{a}^{\prime}}\right) \in \mathbf{V}_{j}^{p^{\prime}}\left(\omega^{\mathbf{a}, \mathbf{a}^{\prime}}\right) \times Q_{j}^{p^{\prime}}\left(\omega^{\mathbf{a}, \mathbf{a}^{\prime}}\right)$ such that

$$
\begin{aligned}
& \left(\mathbf{A}^{-1} \boldsymbol{\sigma}_{j, \mathrm{alg}}^{\mathbf{a}, \mathbf{a}^{\prime}}, \mathbf{v}_{j}\right)_{\omega^{\mathbf{a}, \mathbf{a}^{\prime}}}-\left(\gamma_{j}^{\mathbf{a}, \mathbf{a}^{\prime}}, \nabla \cdot \mathbf{v}_{j}\right)_{\omega^{\mathbf{a}, \mathbf{a}^{\prime}}}=0 \quad \forall \mathbf{v}_{j} \in \mathbf{V}_{j}^{p^{\prime}}\left(\omega^{\mathbf{a}, \mathbf{a}^{\prime}}\right), \\
& \left(\nabla \cdot \boldsymbol{\sigma}_{j, \mathrm{alg}}^{\mathbf{a}, \mathbf{a}^{\prime}}, q_{j}\right)_{\omega^{\mathbf{a}, \mathbf{a}^{\prime}}}=\left(g^{I I, \mathbf{a}, \mathbf{a}^{\prime}, j}, q_{j}\right)_{\omega^{\mathbf{a}, \mathbf{a}^{\prime}}} \quad \forall q_{j} \in Q_{j}^{p^{\prime}}\left(\omega^{\mathbf{a}, \mathbf{a}^{\prime}}\right) .
\end{aligned}
$$

Analogously to (5.3) and (5.5), an equivalent formulation of problem (6.13) is

$$
\sigma_{j, \text { alg }}^{\mathbf{a}, \mathbf{a}^{\prime}}=\underset{\substack{\mathbf{v}_{j} \in \mathbf{V}_{j}^{p^{\prime}}\left(\omega^{\mathbf{a}, \mathbf{a}^{\prime}}\right) \\ \nabla \cdot \mathbf{v}_{j}=g^{I l, \mathbf{a}^{\prime}, j}}}{\arg \min }\left\|\mathbf{A}^{-1 / 2} \mathbf{v}_{j}\right\|_{\omega^{\mathbf{a}, \mathbf{a}^{\prime}}} .
$$

Construction 6.1 supplemented with the above Construction 6.3 is presented in an algorithmic form in Algorithm 2.

\section{$7 \mathrm{H}(\operatorname{div}, \Omega)$-lifting of a given piecewise polynomial with single element solves}

This section presents a construction of a local lifting $\sigma_{j, \text { alg }}^{\mathbf{a}, \mathbf{a}^{\prime}}$ satisfying (6.7) that is yet cheaper than Construction 6.3. It produces

$$
\begin{aligned}
\boldsymbol{\sigma}_{h, \text { alg }} \in \mathbf{V}_{J}^{p}, \nabla \cdot \boldsymbol{\sigma}_{h, \text { alg }}=r_{h}, & \text { contributions to }\left\|\mathbf{A}^{-1 / 2} \boldsymbol{\sigma}_{h, \text { alg }}\right\| \text { are locally minimized } \\
& \text { with degree } p \text { on single elements }(j=J) \\
& \text { and explicitely prescibed with degree } 0(j<J) \\
& \text { by sweeps of Figures } 5,6 .
\end{aligned}
$$

As in (5.1) and (6.1), it thus ensures $\boldsymbol{\sigma}_{h, \text { alg }} \in \mathbf{V}_{J}^{p}$ and $\nabla \cdot \boldsymbol{\sigma}_{h, \mathrm{alg}}=r_{h}$, but it relies on a sequential sweep through the elements of each small patch to further reduce the computational burden, in the spirit of the equilibration procedures in [13], [23], [11], [22, Sec. 7.3], [16, Sec. 5.1], [14, Secs. 3 and 5]. 


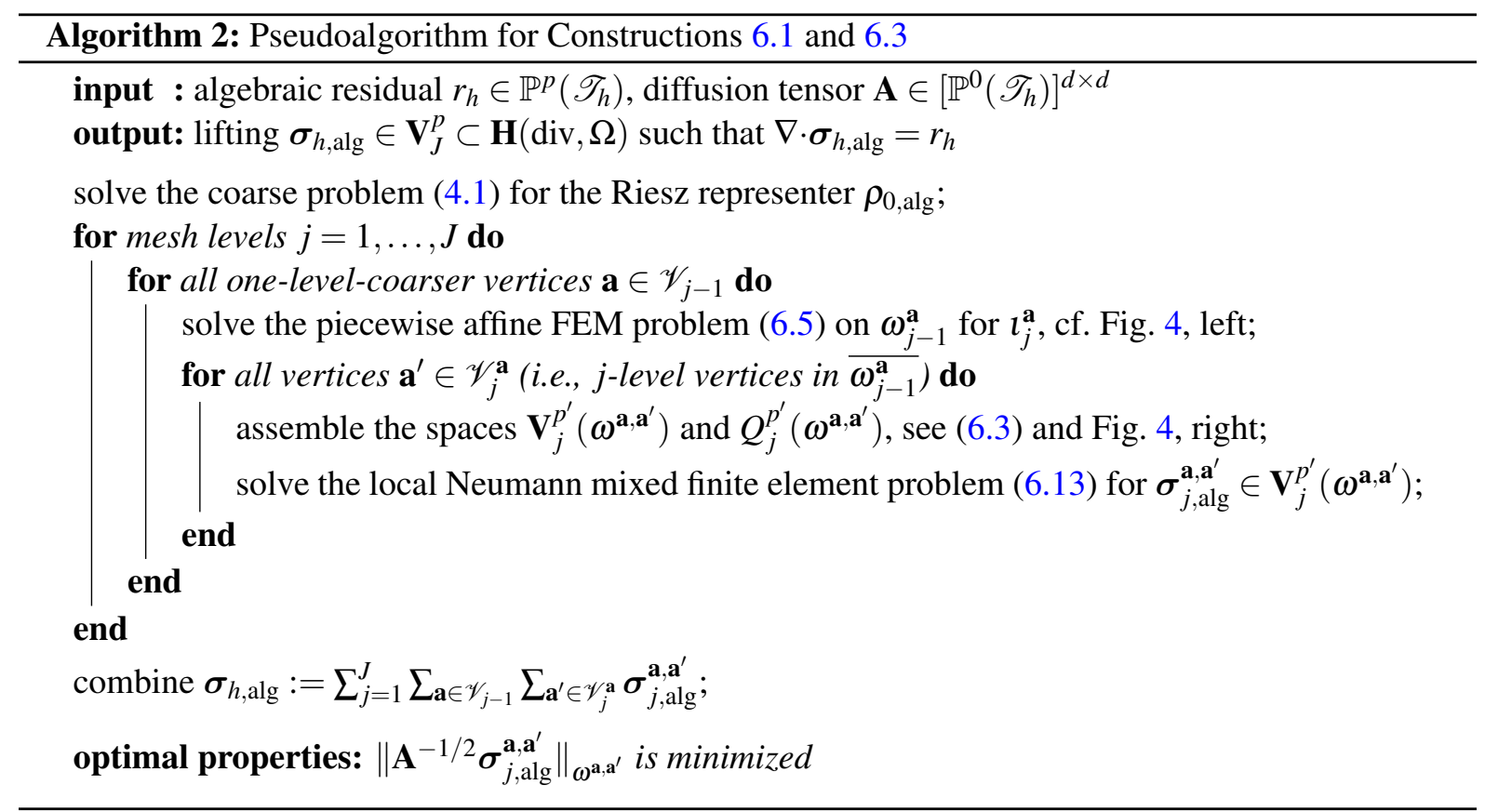

Construction 7.1 (Local lifting $\boldsymbol{\sigma}_{j, \text { alg }}^{\mathbf{a}, \mathbf{a}^{\prime}}$ by a sweep over each small patch and single element solves). Let $1 \leq j \leq J, \mathbf{a} \in \mathscr{V}_{j-1}$, and $\mathbf{a}^{\prime} \in \mathscr{V}_{j}^{\mathbf{a}}$ be given. Set $p^{\prime}:=0$ for $1 \leq j<J$ and $p^{\prime}:=p$ for $j=J$. Let $\left.g^{I I, \mathbf{a}, \mathbf{a}^{\prime}, j} \in \mathbb{P}^{p^{\prime}}\left(\mathscr{T}_{j}\right)\right|_{\omega^{\mathbf{a}, \mathbf{a}^{\prime}}}$ be given by (6.8). Let the simplices in the patch subdomain $\omega^{\mathbf{a}, \mathbf{a}^{\prime}}$ be ordered as $K_{1}, K_{2}, \ldots, K_{M}$ such that $K_{i}$ and $K_{i-1}$ share a common $(d-1)$-dimensional face, $1<i \leq M$ (a suitable ordering in three space dimensions is given in [16, Lemma B.1]). Then, for $1 \leq i \leq M$ :

1. Find $\boldsymbol{\sigma}_{i} \in \mathbf{R T N}^{p^{\prime}}\left(K_{i}\right)=\left\{\mathbf{v} \in\left[\mathbb{P}^{p^{\prime}}\left(K_{i}\right)\right]^{d}+\mathbb{P}^{p^{\prime}}\left(K_{i}\right) \mathbf{x}\right\}$ such that

$$
\boldsymbol{\sigma}_{i}:=\underset{\mathbf{v}_{j} \in \widetilde{\mathbf{R T N}}\left(K_{i}\right)}{\arg \min }\left\|\mathbf{A}^{-1 / 2} \mathbf{v}_{j}\right\|_{K_{i}}
$$

where $\widetilde{\mathbf{R T N}}\left(K_{i}\right)$ are functions of $\mathbf{R T N}^{p^{\prime}}\left(K_{i}\right)$ such that

$$
\begin{aligned}
& \nabla \cdot \mathbf{v}_{j}=g^{I I, \mathbf{a}, \mathbf{a}^{\prime}, j}, \\
& \mathbf{v}_{j} \cdot \mathbf{n}_{F}=\left\{\begin{array}{cl}
\boldsymbol{\sigma}_{j, \text { alg }}^{\mathbf{a}, \mathbf{a}^{\prime}} \cdot \mathbf{n}_{F} & \text { for } F \subset \partial K_{i} \cap \partial K_{\ell}, \quad \ell<i, \\
0 & \text { for } F \subset \partial K_{i} \cap \partial \omega^{\mathbf{a}, \mathbf{a}^{\prime}}, \quad \text { if } \mathbf{a} \in \mathscr{V}_{j-1}^{\text {int }}, \\
0 & \text { for } F \subset \partial K_{i} \cap \partial \omega^{\mathbf{a}, \mathbf{a}^{\prime}}, \quad \text { if } \mathbf{a} \in \mathscr{V}_{j-1}^{\text {ext }} \wedge \mathbf{a}^{\prime} \in \mathscr{V}_{j}^{\text {int }}, \\
0 & \text { for } F \subset \partial K_{i} \cap\left(\partial \omega^{\mathbf{a}, \mathbf{a}^{\prime}} \backslash \partial \Omega\right), \quad \text { if } \mathbf{a} \in \mathscr{V}_{j-1}^{\text {ext }} \wedge \mathbf{a}^{\prime} \in \mathscr{V}_{j}^{\text {ext }} .
\end{array}\right.
\end{aligned}
$$

Here $F$ denotes a $(d-1)$-dimensional face and $\mathbf{n}_{F}$ the associated unit normal vector, whose orientation is chosen arbitrarily but fixed for interior faces and coinciding with the exterior normal of $\Omega$ for exterior faces. An illustration is given in Fig. 5 for $\mathbf{a} \in \mathscr{V}_{j-1}^{\text {int }}$ and in Fig. 6 for $\mathbf{a} \in \mathscr{V}_{j-1}^{\text {ext }}$.

2. Set $\left.\boldsymbol{\sigma}_{j, \text { alg }}^{\mathbf{a},{ }^{\prime}}\right|_{K_{i}}:=\boldsymbol{\sigma}_{i}$.

Constructions 6.1 with 7.1 are presented in an algorithmic form in Algorithm 3. 


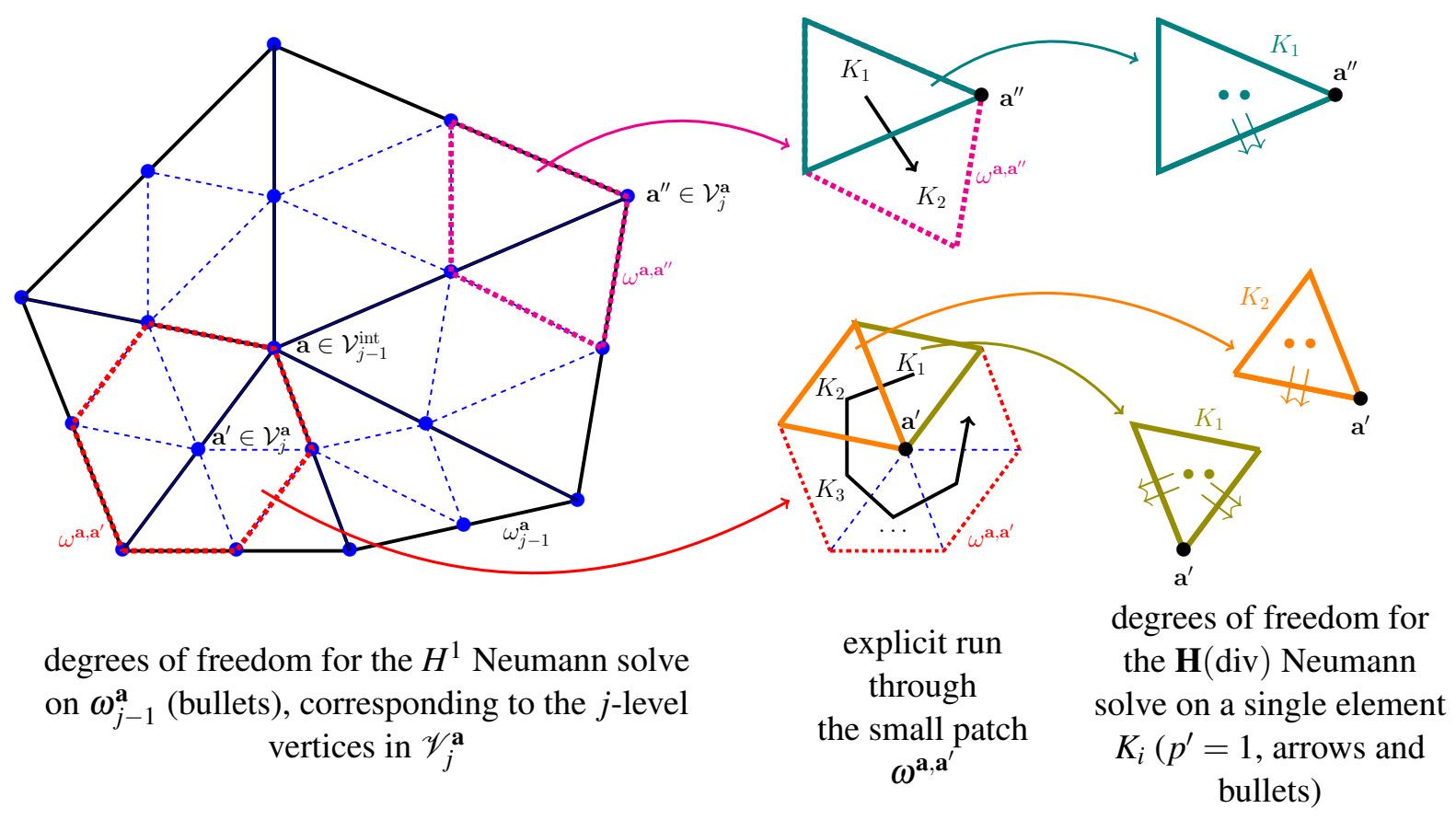

Figure 5: $\mathbf{H}(\operatorname{div}, \Omega)$-lifting of Construction 6.1 and Construction 7.1 by piecewise affine conforming FEM problem on the large patch subdomain $\omega_{j-1}^{\mathrm{a}}$, a sequential sweep through the elements of small patches $\omega^{\mathbf{a}, \mathbf{a}^{\prime}}, \mathbf{a}^{\prime} \in \mathscr{V}_{j}^{\mathbf{a}}$, and single element RTN solve on $K_{i} \subset \overline{\omega^{\mathbf{a}, \mathbf{a}^{\prime}}} ;$ interior vertex $\mathbf{a} \in \mathscr{V}_{j-1}^{\text {int }}$

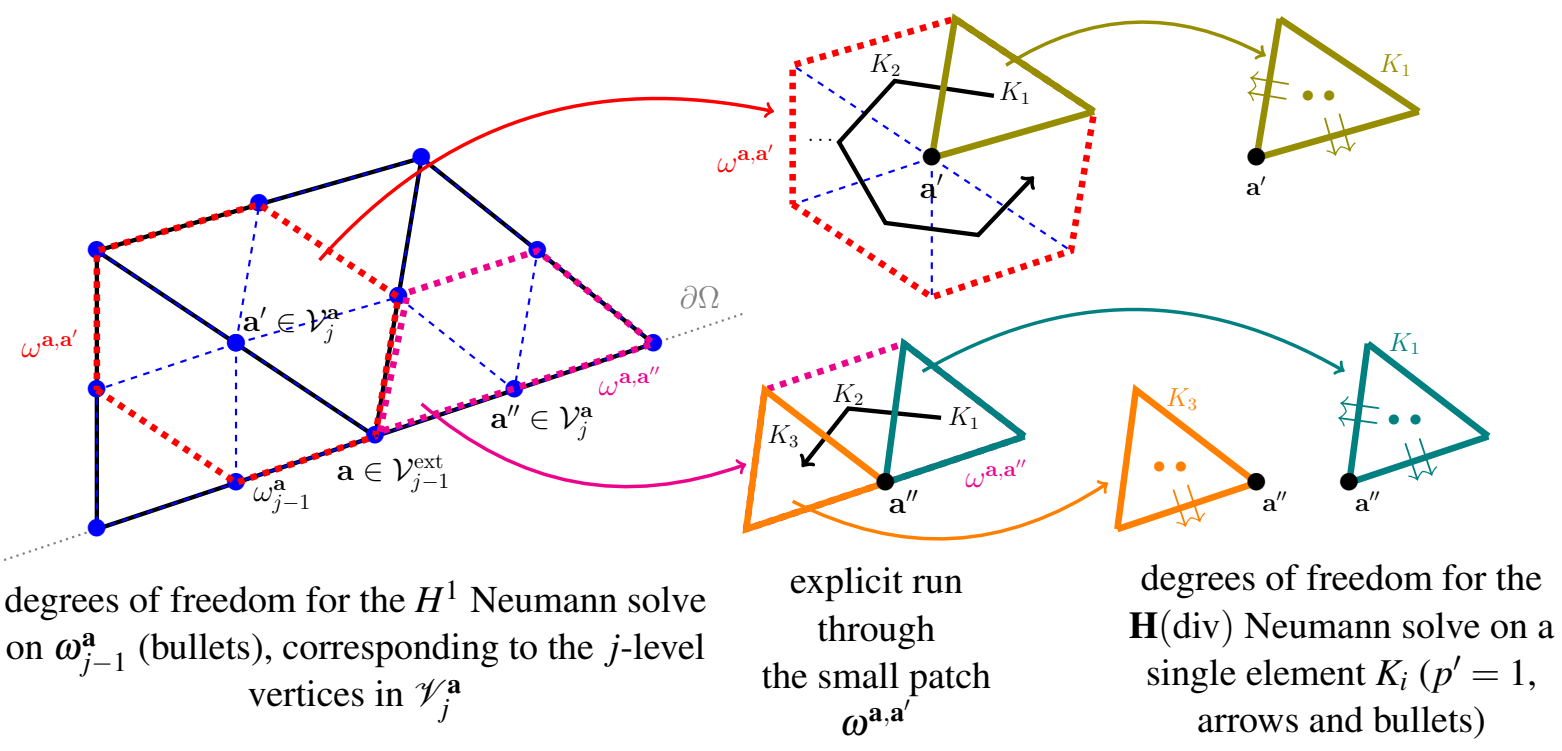

Figure 6: $\mathbf{H}(\operatorname{div}, \Omega)$-lifting of Construction 6.1 and Construction 7.1 by piecewise affine conforming FEM problem on the large patch subdomain $\omega_{j-1}^{\mathrm{a}}$, a sequential sweep through the elements of small patches $\omega^{\mathbf{a}, \mathbf{a}^{\prime}}, \mathbf{a}^{\prime} \in \mathscr{V}_{j}^{\mathbf{a}}$, and single element RTN solve on $K_{i} \subset \overline{\omega^{\mathbf{a}, \mathbf{a}^{\prime}}} ;$ boundary vertex $\mathbf{a} \in \mathscr{V}_{j-1}^{\text {ext }}$ 


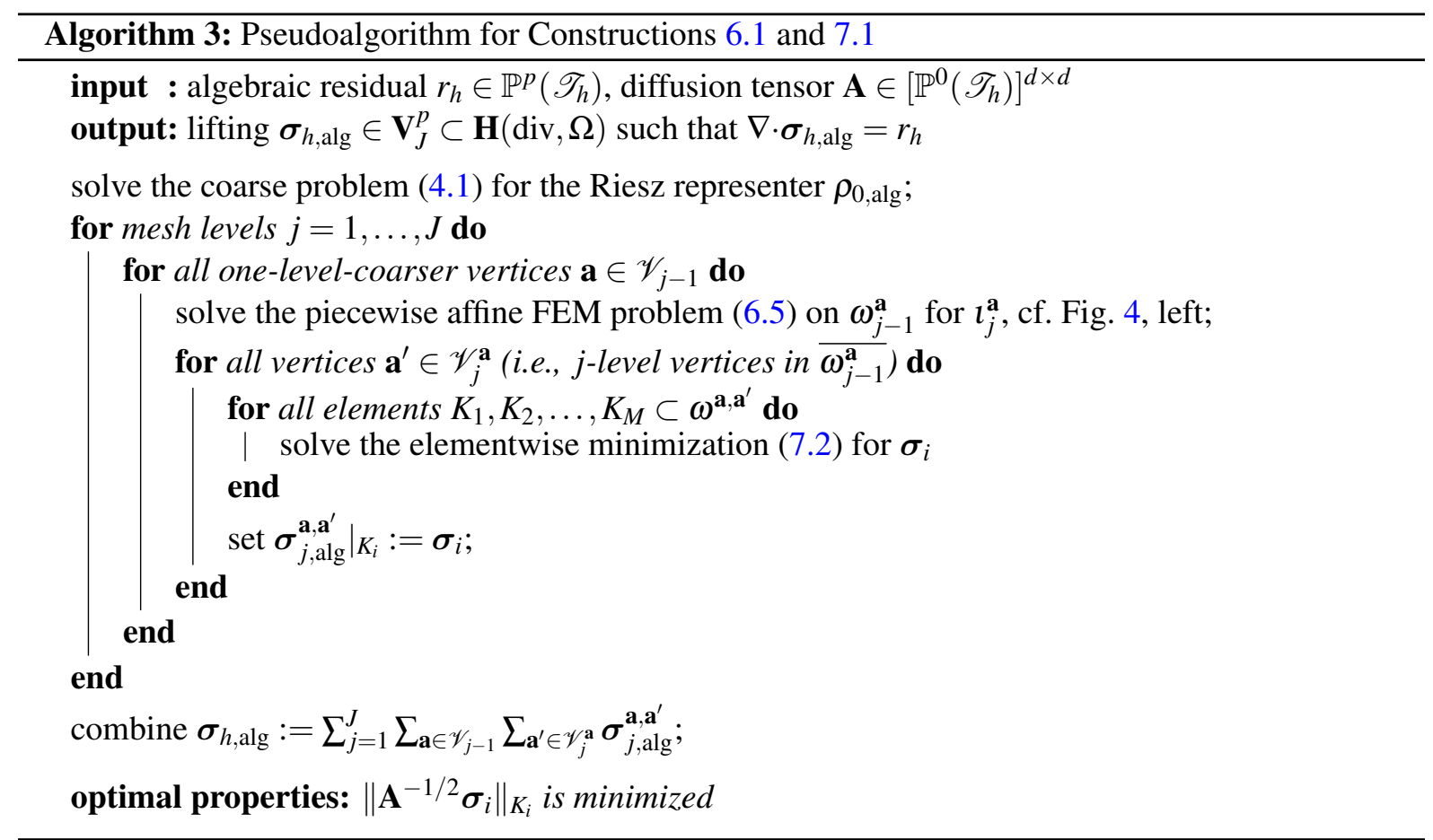

Remark 7.1 (Explicit prescription for $p^{\prime}=0$ ). For $p^{\prime}=0$ in two space dimensions, aside from the boundary $\partial \Omega$, the dimension of the local space $\widetilde{\mathbf{R T N}}\left(K_{i}\right)$ in (7.2) is equal to one on all but the first element $K_{1}$ and the last element $K_{M}$, where it is respectively equal to 2 and 0 . In these cases, Construction 7.1 thus gives a formula that is explicit without any need for solving an algebraic system. Following [16, Sec. 5.1], actually, all the above systems (7.2) in the lowest-degree RTN space with $p^{\prime}=0$ can be replaced by an explicit prescription without any impact on theoretical properties, whence the simplified description written as "explicit" in (7.1) and (8.1).

\section{Approximate lifting in the lowest-degree RTN space}

In Construction 7.1, one can consider setting $p^{\prime}=0$ also for the finest mesh level $j=J$. Denoting the corresponding output in (6.9) by $\sigma_{h, 0, \text { alg }}$, this leads to

$$
\begin{array}{r}
\boldsymbol{\sigma}_{h, 0, \text { alg }} \in \mathbf{V}_{J}^{0}, \nabla \cdot \boldsymbol{\sigma}_{h, 0, \text { alg }}=\Pi_{J}^{0} r_{h}, \text { contributions to } \boldsymbol{\sigma}_{h, 0, \text { alg }} \text { are explicitely prescribed } \\
\text { with degree } 0(j \leq J) \text { by sweeps of Figures } 5,6 .
\end{array}
$$

This procedure is yet much cheaper, since the lowest-degree RTN functions are used also on the finest level $J$; this becomes in particular striking in the illustrative comparison in Sec. 9 below. The price to pay is that $\nabla \cdot \sigma_{h, 0, \text { alg }}$ only equals to the mean value of $r_{h}$ on each mesh element $K \in \mathscr{T}_{h}$, i.e., we only have

$$
\nabla \cdot \boldsymbol{\sigma}_{h, 0, \mathrm{alg}}=\Pi_{J}^{0} r_{h}
$$

in place of $\nabla \cdot \boldsymbol{\sigma}_{h, \text { alg }}=r_{h}$ present in all (5.1), (6.1), and (7.1). Recall the notation $\mathbf{v}_{F}$ for the lowestdegree RTN basis function associated with a $(d-1)$-dimensional face $F$ from (3.2). Let $\mathscr{F}_{K}$ collect all the $(d-1)$-dimensional faces of a mesh element $K$. This construction is presented in an algorithmic form in Algorithm 4. 


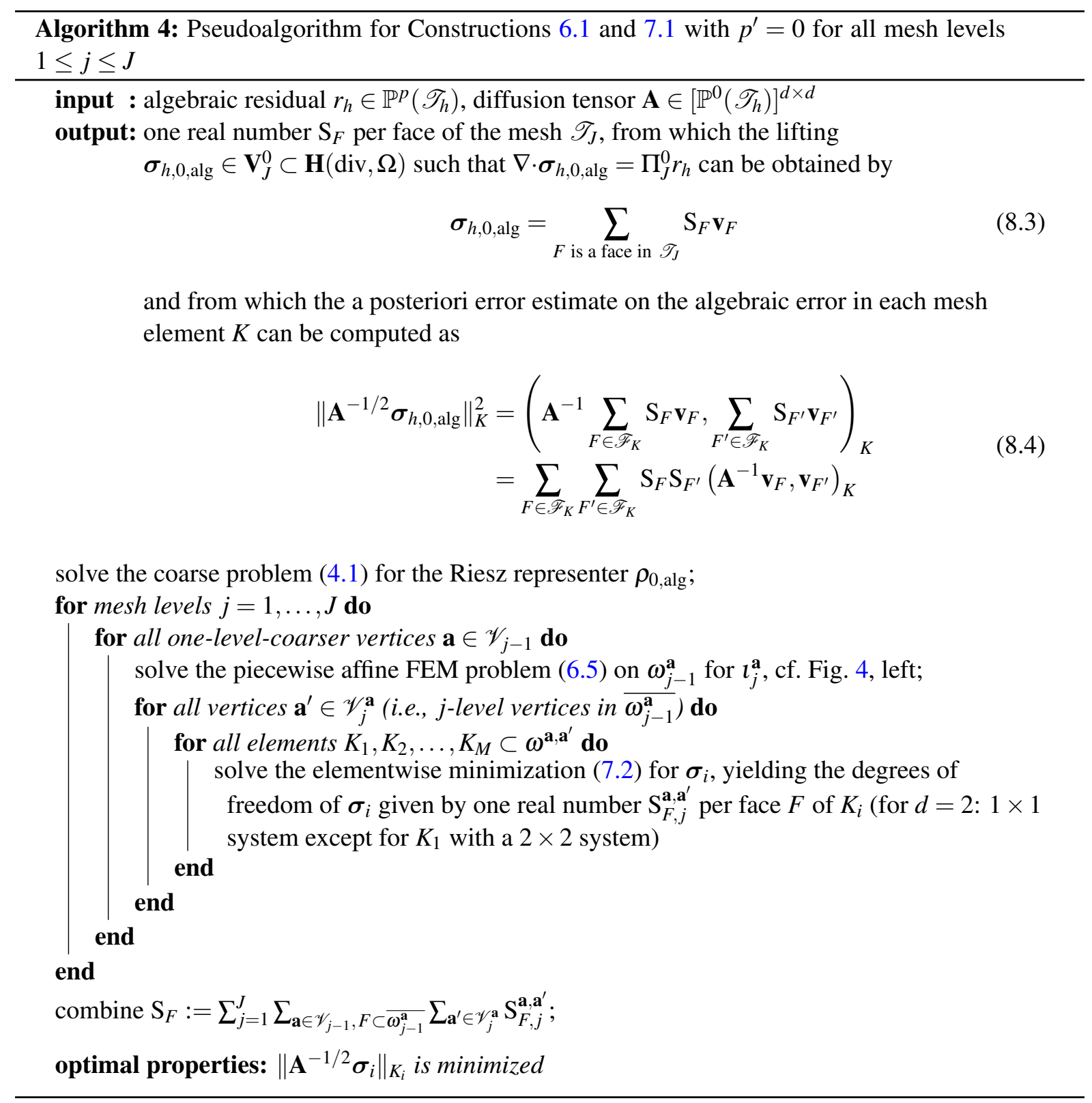


Remark 8.1 (Cheap implementation). Algorithm 4 suggests a very cheap implementation of our estimators. It in particular does not request the computer implementation of the RTN spaces. Indeed, (8.3) follows by developing $\boldsymbol{\sigma}_{h, 0, \mathrm{alg}}$ in the basis $\mathbf{v}_{F}$ on level $J$, recalling (3.2), and the a posteriori error estimate on the algebraic error $\left\|\mathbf{A}^{-1 / 2} \sigma_{h, 0, a l g}\right\|_{K}$ is analytically computable using the closed-form expression (8.4).

Remark 8.2 (Variants). It is also possible to construct the lowest-degree approximate $\mathbf{H}(\operatorname{div}, \Omega)$-liftings analogously to Construction 5.1 or Constructions 6.1 and 6.3. For the sake of simplicity, we do not consider such liftings in this manuscript.

\section{Comparison of the sizes of the local problems in the constructions}

In this section we compare the sizes of the local problems that are solved in the construction of the liftings presented above. For the purpose of this section, we evaluate the number and the sizes of the local problems for one fixed inner patch subdomain $\omega_{j-1}^{\mathrm{a}}$ in two space dimensions, $d=2$, depicted in Figures 3, 4, and 5. This patch subdomain $\omega_{j-1}^{\mathrm{a}}$ consists of 24 elements, 19 vertices (12 on the boundary of $\omega_{j-1}^{\mathbf{a}}, 7$ in the interior of $\left.\omega_{j-1}^{\mathbf{a}}\right), 42$ edges (12 on the boundary of $\omega_{j-1}^{\mathbf{a}}, 30$ in the interior of $\omega_{j-1}^{\mathbf{a}}$ ). Below we use the fact that the dimension of $p$-degree polynomials on a triangle $K \operatorname{dim}\left(\mathbb{P}^{p}(K)\right)$ equals to $(p+1)(p+2) / 2$ in two space dimensions, $d=2$.

\subsection{Finest level $j=J$}

Consider first the finest level $j=J$. We discuss separately the different constructions, remarking that the constructions described in Secs. 6-8 first solve problem (6.5) with the size equal to the number of all vertices in $\omega_{j-1}^{\mathbf{a}}$ minus one (for the mean value condition), that is 18 .

\section{Lifting of Section 5}

For the lifting given by Construction 5.1, there are, in the Raviart-Thomas spaces, $p(p+1)$ degrees of freedom for each element and $p+1$ for each inner edge, see [10, Section 2.3.1]. This yields

$$
\operatorname{dim}\left(\mathbf{V}_{j}^{p}\left(\omega_{j-1}^{\mathbf{a}}\right)\right)=24 p(p+1)+30(p+1),
$$

so that the size of the local Neumann problem (5.3) is

$$
\begin{aligned}
\operatorname{dim}\left(\mathbf{V}_{j}^{p}\left(\omega_{j-1}^{\mathbf{a}}\right)\right)+\operatorname{dim} & \left(Q_{j}^{p}\left(\omega_{j-1}^{\mathbf{a}}\right)\right) \\
& =[24 p(p+1)+30(p+1)]+\left[24 \frac{(p+1)(p+2)}{2}-1\right]=36 p^{2}+90 p+53 .
\end{aligned}
$$

\section{Lifting of Section 6}

The lifting given by Constructions 6.1 and 6.3 requires the solution of local Neumann problems (6.13) on subpatches $\omega^{\mathbf{a}, \mathbf{a}^{\prime}}$. There are 7 inner subpatches (for each inner vertex of patch subdomain $\omega_{j-1}^{\mathbf{a}}$ ) and 12 subpatches on the boundary of $\omega_{j-1}^{\mathbf{a}}$, out of which 6 have 2 elements and 6 have 3 elements. Then we have

$$
\operatorname{dim}\left(\mathbf{V}_{j}^{p}\left(\omega^{\mathbf{a}, \mathbf{a}^{\prime}}\right)\right)=\left\{\begin{array}{cl}
6(p+1)+6 p(p+1) & \text { for an inner subpatch } \omega^{\mathbf{a}, \mathbf{a}^{\prime}} \text { with } 6 \mathrm{el} . \\
p+1+2 p(p+1) & \text { for a boundary subpatch } \omega^{\mathbf{a}, \mathbf{a}^{\prime}} \text { with } 2 \mathrm{el} . \\
2(p+1)+3 p(p+1) & \text { for a boundary subpatch } \omega^{\mathbf{a}, \mathbf{a}^{\prime}} \text { with } 3 \mathrm{el} .
\end{array}\right.
$$

The associated dimensions of $Q_{j}^{p}\left(\omega^{\mathbf{a}, \mathbf{a}^{\prime}}\right)$ are $6(p+1)(p+2) / 2-1,2(p+1)(p+2) / 2$, and $3(p+1)(p+2) / 2$, respectively. 
To sum up, for the lifting given by Constructions 6.1 and 6.3, we solve

- one problem of size 18 ,

- 7 problems of size $9 p^{2}+21 p+11$,

- 6 problems of size $3 p^{2}+6 p+3$,

- 6 problems of size $9 p^{2} / 2+19 p / 2+5$.

\section{Lifting of Section 7}

Constructions 6.1 and 7.1 involve a sweep over elements, see Figure 5 for an illustration, and the solve of elementwise Neumann problems corresponding to (7.2). The dimensions of the local spaces $\widetilde{\mathbf{R T N}}\left(K_{i}\right)$ are

$$
\operatorname{dim}\left(\widetilde{\mathbf{R T N}}\left(K_{i}\right)\right)=\left\{\begin{array}{cl}
2(p+1)+p(p+1) & \text { for } K_{1} \text { of an inner subpatch } \\
p(p+1) & \text { for the last el. of all subpatches } \\
(p+1)+p(p+1) & \text { for all the other el. }
\end{array}\right.
$$

The local spaces for verifying the prescribed divergence of $\widetilde{\mathbf{R T N}}\left(K_{i}\right)$ functions (as e.g. in (6.13b)) are of sizes $(p+1)(p+2) / 2,(p+1)(p+2) / 2-1$, and $(p+1)(p+2) / 2$, respectively. Therefore, for the lifting given by Constructions 6.1 and 7.1 , we solve

- one problem of size 18 ,

- 7 problems of size $3 p^{2} / 2+9 p / 2+3$,

- $7+6+6$ problems of size $3 p^{2} / 2+5 p / 2$,

- $7 \times 4+6 \times 1+6 \times 2$ problems of size $3 p^{2} / 2+7 p / 2+2$.

\section{Lifting of Section 8}

Finally, the construction of Sec. 8 , based on lowest-degree polynomials, requires only solution of algebraic systems $2 \times 2$ on the first elements of inner subpatches. Therefore, we solve

- one problem of size 18 ,

- 7 problems of size 2 .

Importantly, these sizes do not depend on $p$ here. Moreover, all the $2 \times 2$ problems can also be replaced by a direct prescription, see Remark 7.1, so that one is potentially only left with one problem of size 18 .

To provide a more concrete insight, we give in Table 1 a comparison of number of operations for the various constructions and polynomial degrees $p=1,2,3,4$, assuming for simplicity that the solution of an algebraic system of size $n$ takes $n^{3} / 3$ operations. We note that these numbers do not count the operations needed for assembling the local matrices and right-hand sides. As such counts are very implementation dependent, we do not discuss them here.

\begin{tabular}{c|cccc}
$p$ & Constr. 5.1 & Constr. 6.1 and 6.3 & Constr. 6.1 and 7.1 & RTN0 Constr. \\
\hline 1 & $1.8 \mathrm{e}+06$ & $1.8 \mathrm{e}+05$ & $8.9 \mathrm{e}+03$ & $2.0 \mathrm{e}+03$ \\
2 & $1.7 \mathrm{e}+07$ & $1.8 \mathrm{e}+06$ & $7.1 \mathrm{e}+04$ & $2.0 \mathrm{e}+03$ \\
3 & $8.9 \mathrm{e}+07$ & $9.7 \mathrm{e}+06$ & $3.7 \mathrm{e}+05$ & $2.0 \mathrm{e}+03$ \\
4 & $3.2 \mathrm{e}+08$ & $3.6 \mathrm{e}+07$ & $1.4 \mathrm{e}+06$ & $2.0 \mathrm{e}+03$
\end{tabular}

Table 1: Estimated number of operations needed for the solution of the algebraic systems in the constructions on an interior patch subdomain $\omega_{j-1}^{\mathbf{a}}$ on the finest level $j=J$. 


\subsection{Intermediate levels $1 \leq j<J$}

Other important savings are on the intermediate levels, where the new constructions proposed in this paper use zero-degree piecewise RTN functions in place of $p$-degree piecewise RTN functions. We summarize the results, which are naturally more contrasting, in Table 2.

\begin{tabular}{c|cccc}
$p$ & Constr. 5.1 & Constr. 6.1 and 6.3 & Constr. 6.1 and 7.1 & RTN0 Constr. \\
\hline 1 & $1.8 \mathrm{e}+06$ & $5.1 \mathrm{e}+03$ & $2.0 \mathrm{e}+03$ & $2.0 \mathrm{e}+03$ \\
2 & $1.7 \mathrm{e}+07$ & $5.1 \mathrm{e}+03$ & $2.0 \mathrm{e}+03$ & $2.0 \mathrm{e}+03$ \\
3 & $8.9 \mathrm{e}+07$ & $5.1 \mathrm{e}+03$ & $2.0 \mathrm{e}+03$ & $2.0 \mathrm{e}+03$ \\
4 & $3.2 \mathrm{e}+08$ & $5.1 \mathrm{e}+03$ & $2.0 \mathrm{e}+03$ & $2.0 \mathrm{e}+03$
\end{tabular}

Table 2: Estimated number of operations needed for the solution of the algebraic systems in the constructions on an interior patch subdomain $\omega_{j-1}^{\mathrm{a}}$ on intermediate levels $1 \leq j<J$.

\section{Guaranteed upper bounds on the algebraic error in the diffusion prob- lem}

We now consider a finite element discretization of a model diffusion problem and the solution of the arising system of linear algebraic equations by an arbitrary solver. We present the notion of the algebraic residual and derive guaranteed upper bounds on the algebraic error that are based on the liftings presented in the previous sections.

\subsection{Model problem}

Given a source term $f \in L^{2}(\Omega)$ and a bounded symmetric positive definite piecewise constant tensor $\mathbf{A} \in\left[\mathbb{P}^{0}\left(\mathscr{T}_{h}\right)\right]^{d \times d}$, the model diffusion problem reads: find $u \in H_{0}^{1}(\Omega)$ such that

$$
(\mathbf{A} \nabla u, \nabla v)=(f, v) \quad \forall v \in H_{0}^{1}(\Omega) .
$$

The Galerkin finite element approximation of (10.1) consists in finding $u_{h}^{\mathrm{ex}} \in V_{h}^{p}$ such that

$$
\left(\mathbf{A} \nabla u_{h}^{\mathrm{ex}}, \nabla v_{h}\right)=\left(f, v_{h}\right) \quad \forall v_{h} \in V_{h}^{p},
$$

where we recall from Sec. 3.2 that $V_{h}^{p}=\mathbb{P}^{p}\left(\mathscr{T}_{h}\right) \cap H_{0}^{1}(\Omega)=V_{J}^{p}$. Let $\psi_{h}^{l}, 1 \leq l \leq N_{h}, N_{h}:=\operatorname{dim} V_{h}^{p}$, form a basis of the space $V_{h}^{p}$. Then problem (10.2) is equivalent to solving a system of linear algebraic equations with a symmetric positive definite matrix: find $\mathrm{U}_{h}^{\mathrm{ex}} \in \mathbb{R}^{N_{h}}$ such that

$$
\mathbb{A}_{h} \mathrm{U}_{h}^{\mathrm{ex}}=\mathrm{F}_{h}
$$

where $\left(\mathbb{A}_{h}\right)_{l m}:=\left(\mathbf{A} \nabla \psi_{h}^{m}, \nabla \psi_{h}^{l}\right),\left(\mathrm{F}_{h}\right)_{l}:=\left(f, \psi_{h}^{l}\right)$, and $u_{h}^{\mathrm{ex}}=\sum_{m=1}^{N_{h}}\left(\mathrm{U}_{h}^{\mathrm{ex}}\right)_{m} \psi_{h}^{m}$. We note that the algebraic vector $\mathrm{U}_{h}^{\text {ex }}$ depends on the choice of the basis of $V_{h}^{p}$, while the piecewise polynomial function $u_{h}^{\mathrm{ex}} \in V_{h}^{p}$ does not.

\subsection{Algebraic residual}

Let $\mathrm{U}_{h} \in \mathbb{R}^{N_{h}}$ be an arbitrary approximation to the exact solution $\mathrm{U}_{h}^{\mathrm{ex}}$ of (10.3), corresponding to

$$
u_{h}=\sum_{m=1}^{N_{h}}\left(\mathrm{U}_{h}\right)_{m} \psi_{h}^{m} \in V_{h}^{p}
$$


The algebraic residual vector is then

$$
\mathrm{R}_{h}:=\mathrm{F}_{h}-\mathbb{A}_{h} \mathrm{U}_{h} .
$$

Following Papež et al. [29], we associate with $\mathrm{R}_{h}$ a discontinuous elementwise polynomial $r_{h}$ of degree $p$, vanishing on the boundary of $\Omega$, i.e., $r_{h} \in \mathbb{P}^{p}\left(\mathscr{T}_{h}\right),\left.r_{h}\right|_{\partial \Omega}=0$. Denote by supp $\psi_{h}^{l}$ the support of the basis function $\psi_{h}^{l}$. Then, for each fixed element $K \in \mathscr{T}_{h}$, we define $\left.r_{h}\right|_{K} \in \mathbb{P}^{p}(K)$ by

$$
\left(r_{h}, \psi_{h}^{l}\right)_{K}=\left(\mathrm{R}_{h}\right)_{l} \frac{|K|}{\left|\operatorname{supp} \psi_{h}^{l}\right|},\left.\quad r_{h}\right|_{\partial K \cap \partial \Omega}=0,
$$

for all basis functions $\psi_{h}^{l}$ of the space $V_{h}^{p}$ non-vanishing on $K$. Such $r_{h}$ satisfies obviously $\left(\mathrm{R}_{h}\right)_{l}=$ $\left(r_{h}, \psi_{h}^{l}\right), 1 \leq l \leq N_{h}$, and the algebraic relation (10.5) yields

$$
\left(r_{h}, v_{h}\right)=\left(f, v_{h}\right)-\left(\mathbf{A} \nabla u_{h}, \nabla v_{h}\right) \quad \forall v_{h} \in V_{h}^{p} .
$$

We point out that although $r_{h}$ is uniquely defined by (10.6), it is not the unique element in $\mathbb{P}^{p}\left(\mathscr{T}_{h}\right)$ which satisfies (10.7). It is also possible to define $r_{h} \in V_{h}^{p}$ directly by (10.7), as in, e.g., [8]. However, this requires the solution of a globally coupled mass system, see the discussion in [29, Sec. 5.1]. Thus we prefer to work with (10.6) where the construction is local.

Remark 10.1. As discussed in [28, Remark 7.12], the representation $r_{h}$ of the algebraic residual given by (10.6) is specified on the finest mesh $\mathscr{T}_{h}=\mathscr{T}_{J}$ only. The constructions then involve the $L^{2}$-projections (such as $\Pi_{j}^{p^{\prime}}\left(\mathrm{Id}-\Pi_{j-1}^{0}\right)$ in (6.6)) to compute levelwise contributions of the residual.

In the context of hierarchical algebraic solvers, where levelwise algebraic residuals, say $\mathbf{R}_{j}$, can be evaluated, one can proceed alternatively. Analogously to (10.6) we can define the representations of the algebraic residual $\mathrm{R}_{j}$ on each level, i.e. $r_{j} \in \mathbb{P}^{p}\left(\mathscr{T}_{j}\right),\left.r_{j}\right|_{\partial \Omega}=0$, satisfying

$$
\left(r_{j}, v_{j}\right)=\left(f, v_{j}\right)-\left(\mathbf{A} \nabla u_{h}, \nabla v_{j}\right) \quad \forall v_{j} \in V_{j}^{p} .
$$

Then, we can modify the constructions of the liftings by defining $g^{I, \mathbf{a}, j}$ from (6.6) as follows

$$
g^{I, \mathbf{a}, j}:= \begin{cases}\Pi_{1}^{0}\left(r_{1} \psi_{0}^{\mathbf{a}}-\mathbf{A} \nabla \rho_{0, \mathrm{alg}} \cdot \nabla \psi_{0}^{\mathbf{a}}\right) & j=1, \\ \Pi_{j}^{p^{\prime}}\left(\left(r_{j}-r_{j-1}\right) \psi_{j-1}^{\mathbf{a}}\right) & 1<j \leq J .\end{cases}
$$

Such modified constructions are theoretically superior; following [28, Remark 7.12], we can prove for them efficiency (see Sec. 11 below) with constants depending on the number of mesh levels $J$ at most linearly. Nevertheless, in numerical experiments (described in Sec. 12 below), the differences in the results were negligible with respect to the constructions with $r_{h}$ from (10.6) and $g^{I, \mathbf{a}, j}$ from (6.6). Moreover, the present construction is more general, with one piecewise polynomial input $r_{h} \in \mathbb{P}^{p}\left(\mathscr{T}_{h}\right)$ only.

\subsection{Bounds based on $p$-order lifting of the algebraic residual}

Liftings of the algebraic residual presented in Secs. 5-7 can be used to bound the algebraic error as in [28]:

Theorem 10.1 (Guaranteed bounds on the algebraic error, constructions of Sections 5-7). Let $u_{h} \in V_{h}^{p}$ be arbitrary, let $r_{h} \in \mathbb{P}^{p}\left(\mathscr{T}_{h}\right)$ satisfy (10.7), and let $\boldsymbol{\sigma}_{h, \mathrm{alg}} \in \mathbf{V}_{J}^{p}$ be given by any of the constructions of Sections 5-7. Then

$$
\left\|\mathbf{A}^{1 / 2} \nabla\left(u_{h}^{\mathrm{ex}}-u_{h}\right)\right\| \leq \eta_{\mathrm{alg}}^{p},
$$


where

$$
\begin{array}{ll}
\eta_{\mathrm{alg}}^{p}=\eta_{5.1}^{p}:=\left\|\mathbf{A}^{-1 / 2} \boldsymbol{\sigma}_{h, \mathrm{alg}}\right\| & \text { for } \boldsymbol{\sigma}_{h, \mathrm{alg}} \in \mathbf{V}_{J}^{p} \text { given by Construction 5.1, } \\
\eta_{\mathrm{alg}}^{p}=\eta_{6.3}^{p}:=\left\|\mathbf{A}^{-1 / 2} \boldsymbol{\sigma}_{h, \mathrm{alg}}\right\| & \text { for } \boldsymbol{\sigma}_{h, \mathrm{alg}} \in \mathbf{V}_{J}^{p} \text { given by Constructions 6.1 and 6.3, } \\
\eta_{\mathrm{alg}}^{p}=\eta_{7.1}^{p}:=\left\|\mathbf{A}^{-1 / 2} \boldsymbol{\sigma}_{h, \mathrm{alg}}\right\| & \text { for } \boldsymbol{\sigma}_{h, \mathrm{alg}} \in \mathbf{V}_{J}^{p} \text { given by Constructions 6.1 and 7.1. }
\end{array}
$$

Proof. Using that $\boldsymbol{\sigma}_{h, \mathrm{alg}} \in \mathbf{V}_{J}^{p}$ and $\nabla \cdot \boldsymbol{\sigma}_{h, \mathrm{alg}}=r_{h}$ in all (5.1), (6.1), or (7.1) together with (10.2), (10.7), the Green theorem, and the Cauchy-Schwarz inequality

$$
\begin{aligned}
\left\|\mathbf{A}^{1 / 2} \nabla\left(u_{h}^{\mathrm{ex}}-u_{h}\right)\right\| & =\sup _{v_{h} \in V_{h}^{p},\left\|\mathbf{A}^{1 / 2} \nabla v_{h}\right\|=1}\left(r_{h}, v_{h}\right)=\sup _{v_{h} \in V_{h}^{p},\left\|\mathbf{A}^{1 / 2} \nabla v_{h}\right\|=1}\left(\nabla \cdot \boldsymbol{\sigma}_{h, \text { alg }}, v_{h}\right) \\
& =\sup _{v_{h} \in V_{h}^{p},\left\|\mathbf{A}^{1 / 2} \nabla v_{h}\right\|=1}\left(\boldsymbol{\sigma}_{h, \mathrm{alg}}, \nabla v_{h}\right) \\
& \leq \sup _{v_{h} \in V_{h}^{p},\left\|\mathbf{A}^{1 / 2} \nabla v_{h}\right\|=1}\left\|\mathbf{A}^{-1 / 2} \boldsymbol{\sigma}_{h, \text { alg }}\right\|\left\|\mathbf{A}^{1 / 2} \nabla v_{h}\right\|=\left\|\mathbf{A}^{-1 / 2} \boldsymbol{\sigma}_{h, \text { alg }}\right\| .
\end{aligned}
$$

\subsection{Bound based on the lowest-degree approximate lifting of the algebraic residual}

We now present an upper bound on the algebraic error using the lowest-degree lifting as in Sec. 8. In order to bound the algebraic error, we have to in this case compensate for the fact that $\nabla \cdot \sigma_{h, 0, \text { alg }} \neq r_{h}$, since $\nabla \cdot \sigma_{h, 0, \text { alg }}=\Pi_{J}^{0} r_{h}$ only. One can therefore expect that this bound may deteriorate for approximations using higher polynomial degrees $p$. Let $c_{\mathbf{A}, K}$ denote the smallest eigenvalue of the tensor $\mathbf{A}$ on the mesh element $K$.

Theorem 10.2 (Guaranteed bound on the algebraic error, construction of Sec. 8). Let $u_{h} \in V_{h}^{p}$ be arbitrary, let $r_{h} \in \mathbb{P}^{p}\left(\mathscr{T}_{h}\right)$ satisfy (10.7), and let $\sigma_{h, 0, \text { alg }} \in \mathbf{V}_{J}^{0}$ be given by the construction of Sec. 8. Then

$$
\left\|\mathbf{A}^{1 / 2} \nabla\left(u_{h}^{\mathrm{ex}}-u_{h}\right)\right\| \leq \eta_{\mathrm{alg}}^{0},
$$

where

$$
\begin{aligned}
\eta_{\mathrm{alg}}^{0}:= & \left\{\sum_{K \in \mathscr{T}}\left(\frac{h_{K}}{c_{\mathbf{A}, K}^{1 / 2} \pi}\left\|r_{h}-\Pi_{J}^{0} r_{h}\right\|_{K}+\left\|\mathbf{A}^{-1 / 2} \boldsymbol{\sigma}_{h, 0, \mathrm{alg}}\right\|_{K}\right)^{2}\right\}^{1 / 2}, \\
& \quad \text { for } \boldsymbol{\sigma}_{h, 0, \mathrm{alg}} \in \mathbf{V}_{J}^{0} \text { given by Constructions } 6.1 \text { and } 7.1 \text { with } p^{\prime}=0 \text { for } j=J .
\end{aligned}
$$

Proof. For $v_{h} \in V_{h}^{p}$,

$$
\begin{aligned}
\left(r_{h}, v_{h}\right) & =\left(r_{h}-\Pi_{J}^{0} r_{h}+\Pi_{J}^{0} r_{h}, v_{h}\right) \\
& =\left(r_{h}-\Pi_{J}^{0} r_{h}, v_{h}-\Pi_{J}^{0} v_{h}\right)+\left(\nabla \cdot \boldsymbol{\sigma}_{h, 0, \mathrm{alg}}, v_{h}\right) \\
& \leq \sum_{K \in \mathscr{T}_{h}}\left(\left\|r_{h}-\Pi_{J}^{0} r_{h}\right\|_{K} \frac{h_{K}}{c_{\mathbf{A}, K}^{1 / 2} \pi}\left\|\mathbf{A}^{1 / 2} \nabla v_{h}\right\|_{K}+\left\|\mathbf{A}^{-1 / 2} \boldsymbol{\sigma}_{h, 0, \mathrm{alg}}\right\|_{K}\left\|\mathbf{A}^{1 / 2} \nabla v_{h}\right\|_{K}\right) \\
& \leq\left\{\sum_{K \in \mathscr{T}_{h}}\left(\frac{h_{K}}{c_{\mathbf{A}, K}^{1 / 2} \pi}\left\|r_{h}-\Pi_{J}^{0} r_{h}\right\|_{K}+\left\|\mathbf{A}^{-1 / 2} \boldsymbol{\sigma}_{h, 0, \mathrm{alg}}\right\|_{K}\right)^{2}\right\}^{1 / 2}\left\|\mathbf{A}^{1 / 2} \nabla v_{h}\right\|,
\end{aligned}
$$


where we have used the Poincaré and Cauchy-Schwarz inequalities. Consequently, as in the proof of (10.8),

$$
\begin{aligned}
\left\|\mathbf{A}^{1 / 2} \nabla\left(u_{h}^{\mathrm{ex}}-u_{h}\right)\right\| & =\sup _{v_{h} \in V_{h},\left\|\mathbf{A}^{1 / 2} \nabla v_{h}\right\|=1}\left(r_{h}, v_{h}\right) \\
& \leq\left\{\sum_{K \in \mathscr{T}_{h}}\left(\frac{h_{K}}{c_{\mathbf{A}, K}^{1 / 2} \pi}\left\|r_{h}-\Pi_{J}^{0} r_{h}\right\|_{K}+\left\|\mathbf{A}^{-1 / 2} \boldsymbol{\sigma}_{h, 0, \mathrm{alg}}\right\|_{K}\right)^{2}\right\}^{1 / 2} .
\end{aligned}
$$

\section{Efficiency of the error bounds}

In this section we prove the efficiency of the upper bounds on the algebraic error of Theorems 10.1 and 10.2 , i.e., we show that the bounds overestimate the algebraic error $\left\|\mathbf{A}^{1 / 2} \nabla\left(u_{h}^{\mathrm{ex}}-u_{h}\right)\right\|$ uniformly and not significantly. We start by recalling the efficiency of the upper bound $\eta_{5.1}^{p}$ proved in [28, Theorem 7.4].

Theorem 11.1 (Efficiency of the upper bound $\eta_{5.1}^{p}$ on the algebraic error, [28]). Let $\eta_{5.1}^{p}$ be given by (10.9a). There holds

$$
\eta_{5.1}^{p} \leq \bar{C}_{\text {alg }}^{\text {eff }, p .5 .1}\left\|\mathbf{A}^{1 / 2} \nabla\left(u_{h}^{\mathrm{ex}}-u_{h}\right)\right\|,
$$

where $\bar{C}_{\mathrm{alg}}^{\mathrm{eff}, p, 5.1}$ is a generic constant only depending on the shape regularity of the mesh $\mathscr{T}_{h}$, the space dimension $d$, the polynomial degree $p$, the number of mesh levels $J$, and the ratio of the largest to the smallest eigenvalue of the diffusion coefficient $\mathbf{A}$.

Proof. For later use, we present here the key steps of the proof of [28, Theorem 7.4]. For simplicity of presentation, we consider here $\mathbf{A}$ being the identity matrix; the results for general $\mathbf{A} \in\left[\mathbb{P}^{0}\left(\mathscr{T}_{h}\right)\right]^{d \times d}$ follow easily and give rise to an additional dependence of the constant $C$ below on the ratio of the largest to the smallest eigenvalue of the diffusion coefficient $\mathbf{A}$. Let $C$ be a generic constant only depending on the shape regularity of $\mathscr{T}_{h}, d, p$, and $J$. We denote by $\sigma_{j, 5.1, \text { alg }}^{\mathrm{a}}$ the local contributions of Construction 5.1.

Step 1: Bound the norm of the lifting by the local contributions

$$
\left\|\sigma_{h, \mathrm{alg}}\right\|^{2} \leq J(d+1) \sum_{j=1}^{J} \sum_{\mathbf{a} \in \mathscr{Y} \mathcal{F}_{j-1}}\left\|\sigma_{j, 5.1, \mathrm{alg}}^{\mathrm{a}}\right\|_{\omega_{j-1}^{\mathrm{a}}}^{2} .
$$

Step 2: Bound the local contributions using the norm of the algebraic residual

$$
\begin{array}{rlrl}
\left\|\sigma_{1,5.1, \mathrm{alg}}^{\mathrm{a}}\right\|_{\omega_{0}^{\mathrm{a}}} & \leq C h_{\omega_{0}^{\mathrm{a}}}\left\{\left\|r_{h}\right\|_{\omega_{0}^{\mathrm{a}}}+\left\|\nabla \rho_{0, \mathrm{alg}} \cdot \nabla \psi_{0}^{\mathrm{a}}\right\|_{\omega_{0}^{\mathrm{a}}}\right\}, & \\
\left\|\sigma_{j, 5.1, \mathrm{alg}}^{\mathrm{a}}\right\|_{\omega_{j-1}^{\mathrm{a}}} \leq C h_{\omega_{j-1}^{\mathrm{a}}}\left\|r_{h}\right\|_{\omega_{j-1}^{\mathrm{a}}}, & 1<j \leq J,
\end{array}
$$

where $h_{\omega_{j-1}^{\mathrm{a}}}$ denotes the diameter of the patch $\omega_{j-1}^{\mathrm{a}}$.

Step 3: Bound

$$
h_{K}\left\|r_{h}\right\|_{K} \leq C\left\|\nabla\left(u_{h}^{\mathrm{ex}}-u_{h}\right)\right\|_{\omega_{K}}, \quad \forall K \in \mathscr{T}_{h},
$$

where $\omega_{K}:=\bigcup_{\mathbf{a} \in \mathscr{Y}_{h}, \mathbf{a} \subset \partial K} \omega_{h}^{\mathbf{a}}$ denotes the domain of all elements sharing a node with $K$.

Step 4: Combine Steps 2 and 3 to bound

$$
\sum_{\mathbf{a} \in \mathscr{Y}_{j-1}}\left\|\boldsymbol{\sigma}_{j, 5.1, \mathrm{alg}}^{\mathbf{a}}\right\|_{\omega_{j-1}^{\mathrm{a}}}^{2} \leq C\left\|\nabla\left(u_{h}^{\mathrm{ex}}-u_{h}\right)\right\|^{2}, \quad 1<j \leq J .
$$

Step 5: Combine Steps 2 and 3 together with $\left\|\nabla \rho_{0, \mathrm{alg}}\right\| \leq\left\|\nabla\left(u_{h}^{\mathrm{ex}}-u_{h}\right)\right\|$ to bound

$$
\sum_{\mathbf{a} \in \mathscr{Y}_{0}}\left\|\sigma_{1,5.1, \text { alg }}^{\mathbf{a}}\right\|_{\omega_{0}^{\mathbf{a}}}^{2} \leq C\left\|\nabla\left(u_{h}^{\mathrm{ex}}-u_{h}\right)\right\|^{2} .
$$


Step 6: Combine Steps 1,4, and 5 to prove the assertion of the theorem.

We now prove the efficiency for the upper algebraic error bounds based on the $p$ th-order liftings of the algebraic residual derived in Sections 6 and 7.

Theorem 11.2 (Efficiency of the upper bounds $\eta_{6.3}^{p}$ and $\eta_{7.1}^{p}$ on the algebraic error). Let $\eta_{6.3}^{p}$ and $\eta_{7.1}^{p}$ be respectively given by $(10.9 \mathrm{~b})-(10.9 \mathrm{c})$. There holds

$$
\begin{aligned}
& \eta_{6.3}^{p} \leq \bar{C}_{\mathrm{alg}, p, 6.3}^{\mathrm{e} f}\left\|\mathbf{A}^{1 / 2} \nabla\left(u_{h}^{\mathrm{ex}}-u_{h}\right)\right\|, \\
& \eta_{7.1}^{p} \leq \bar{C}_{\mathrm{alg}}^{\text {eff, } p, 7.1}\left\|\mathbf{A}^{1 / 2} \nabla\left(u_{h}^{\mathrm{ex}}-u_{h}\right)\right\|,
\end{aligned}
$$

where $\bar{C}_{\mathrm{alg}}^{\mathrm{eff}, p, 6.3}, \bar{C}_{\mathrm{alg}, p, 7.1}^{\mathrm{efr}}$ are generic constants only depending on the shape regularity of the mesh $\mathscr{T}_{h}$, the space dimension $d$, the polynomial degree $p$, the number of mesh levels $J$, and the ratio of the largest to the smallest eigenvalue of the diffusion coefficient $\mathbf{A}$.

Proof. We again take here $\mathbf{A}=\mathrm{Id}$ for simplicity of exposition; the general bound follows easily. We show that the norm of the local contributions $\boldsymbol{\sigma}_{j \text {,alg }}^{\mathbf{a}}$ of Construction 6.1, with $\boldsymbol{\sigma}_{j, \text { alg }}^{\mathbf{a}, \mathbf{a}^{\prime}}$ obtained either by Construction 6.3 or 7.1, can be bounded as in the proof of Theorem 11.1 above. For the ease of presentation, we denote by $\boldsymbol{\sigma}_{j, 6.3, \text { alg }}^{\mathbf{a}, \mathbf{a}^{\prime}}$ and by $\boldsymbol{\sigma}_{j, 7.1, \text { alg }}^{\mathbf{a}, \mathbf{a}^{\prime}}$ the local contributions of Construction 6.3 and Construction 7.1, respectively. Let $C$ be a constant only depending on the shape regularity of $\mathscr{T}_{h}$, the space dimension $d$, and the polynomial degree $p$.

Efficiency of $\eta_{6.3}^{p}$ : To start with, let $1<j \leq J$, so that $g^{I, \mathbf{a}, j}$ from (6.6) is given by $\Pi_{j}^{p^{\prime}}\left(\operatorname{Id}-\Pi_{j-1}^{0}\right)\left(r_{h} \psi_{j-1}^{\mathbf{a}}\right)$ for a fixed $\mathbf{a} \in \mathscr{V}_{j-1}$. First,

$$
\left\|g^{I, \mathbf{a}, j}\right\|_{\omega_{j-1}^{\mathrm{a}}}=\left\|\Pi_{j}^{p^{\prime}}\left(\mathrm{Id}-\Pi_{j-1}^{0}\right)\left(r_{h} \psi_{j-1}^{\mathbf{a}}\right)\right\|_{\omega_{j-1}^{\mathrm{a}}} \leq\left\|r_{h} \psi_{j-1}^{\mathrm{a}}\right\|_{\omega_{j-1}^{\mathrm{a}}} \leq\left\|r_{h}\right\|_{\omega_{j-1}^{\mathrm{a}}} .
$$

Second, taking $v_{j}=\imath_{j}^{\mathbf{a}}$ as a test function in (6.5), we find, using the the Poincaré inequality,

$$
\left\|\nabla \mathbf{l}_{j}^{\mathbf{a}}\right\|_{\omega_{j-1}^{\mathrm{a}}}^{2}=\left(g^{I, \mathbf{a}, j}, \imath_{j}^{\mathbf{a}}\right)_{\omega_{j-1}^{\mathrm{a}}} \leq\left\|g^{I, \mathbf{a}, j}\right\|_{\omega_{j-1}^{\mathrm{a}}}\left\|l_{j}^{\mathbf{a}}\right\|_{\omega_{j-1}^{\mathrm{a}}} \leq C h_{\omega_{j-1}^{\mathrm{a}}}\left\|g^{I, \mathbf{a}, j}\right\|_{\omega_{j-1}^{\mathrm{a}}}\left\|\nabla l_{j}^{\mathbf{a}}\right\|_{\omega_{j-1}^{\mathrm{a}}},
$$

so that

$$
\left\|\nabla l_{j}^{\mathbf{a}}\right\|_{\omega_{j-1}^{\mathrm{a}}} \leq C h_{\omega_{j-1}^{\mathrm{a}}}\left\|g^{I, \mathbf{a}, j}\right\|_{\omega_{j-1}^{\mathrm{a}}} .
$$

Third, the inf-sup stability of the mixed finite element discretization (6.13) gives (recall that $\mathbf{A}=\mathrm{Id})$

$$
\left\|\boldsymbol{\sigma}_{j, 6.3, \mathrm{alg}}^{\mathbf{a}, \mathbf{a}^{\prime}}\right\|_{\omega^{\mathbf{a}, \mathbf{a}^{\prime}}} \leq C h_{\omega^{\mathbf{a}, \mathbf{a}^{\prime}}}\left\|g^{I I, \mathbf{a}, \mathbf{a}^{\prime}, j}\right\|_{\omega^{\mathbf{a}, \mathbf{a}^{\prime}}} .
$$

Fourth, using definition (6.8),

$$
\begin{aligned}
\left\|g^{I I, \mathbf{a}, \mathbf{a}^{\prime}, j}\right\|_{\omega^{\mathbf{a}, \mathbf{a}^{\prime}}} & \leq\left\|g^{I, \mathbf{a}, j} \psi_{j}^{\mathbf{a}^{\prime}}\right\|_{\omega^{\mathbf{a}, a^{\prime}}}+\left\|\nabla l_{j}^{\mathbf{a}} \cdot \nabla \psi_{j}^{\mathbf{a}^{\prime}}\right\|_{\omega^{\mathbf{a}, \mathbf{a}^{\prime}}} \\
& \leq\left\|g^{I, \mathbf{a}, j}\right\|_{\omega^{\mathbf{a}, \mathbf{a}^{\prime}}}+C\left\|\nabla l_{j}^{\mathbf{a}}\right\|_{\omega^{\mathbf{a}, \mathbf{a}^{\prime}}} h_{\omega^{\mathbf{a}, \mathbf{a}^{\prime}}}^{-1}
\end{aligned}
$$

Thus, denoting by $\boldsymbol{\sigma}_{j, 6.3, \mathrm{alg}}^{\mathbf{a}}$ the local flux given in (6.9) as a sum of small patch contributions $\boldsymbol{\sigma}_{j, 6.3, \text { alg }}^{\mathbf{a}, \mathbf{a}^{\prime}}$, combining the above results together, we see

$$
\left\|\boldsymbol{\sigma}_{j, 6.3, \mathrm{alg}}^{\mathrm{a}}\right\|_{\omega_{j-1}^{\mathrm{a}}}^{2} \leq C^{2} \sum_{\mathbf{a}^{\prime} \in Y_{j}^{\mathbf{a}}}\left\|\boldsymbol{\sigma}_{j, 6.3, \mathrm{alg}}^{\mathbf{a}, \mathbf{a}^{\prime}}\right\|_{\omega^{\mathrm{a}, \mathrm{a}^{\prime}}}^{2} \leq C^{2} h_{\omega_{j-1}^{\mathrm{a}}}^{2}\left\|r_{h}\right\|_{\omega_{j-1}^{\mathrm{a}}}^{2},
$$

as in (11.1b). 
Let now $j=1$ and $\mathbf{a} \in \mathscr{V}_{0}$. Here $g^{I, \mathbf{a}, 1}=\Pi_{1}^{0}\left(r_{h} \psi_{0}^{\mathbf{a}}\right)-\nabla \rho_{0, \text { alg }} \cdot \nabla \psi_{0}^{\mathbf{a}}$ from (6.6) and we have

$$
\left\|g^{I, \mathbf{a}, 1}\right\|_{\omega_{0}^{\mathbf{a}}} \leq\left\|r_{h}\right\|_{\omega_{0}^{\mathbf{a}}}+\left\|\nabla \rho_{0, \mathrm{alg}} \cdot \nabla \psi_{0}^{\mathbf{a}}\right\|_{\omega_{0}^{\mathbf{a}}}
$$

so that

$$
\left\|\sigma_{1,6.3, \mathrm{alg}}^{\mathbf{a}}\right\|_{\omega_{0}^{\mathbf{a}}}^{2} \leq C^{2} \sum_{\mathbf{a}^{\prime} \in \mathscr{Y}_{1}^{\mathbf{a}}}\left\|\boldsymbol{\sigma}_{1,6.3, \mathrm{alg}}^{\mathbf{a}, \mathbf{a}^{\prime}}\right\|_{\omega^{\mathbf{a}, \mathbf{a}^{\prime}}}^{2} \leq C^{2} h_{\omega_{0}^{\mathbf{a}}}^{2}\left\{\left\|r_{h}\right\|_{\omega_{0}^{\mathbf{a}}}+\left\|\nabla \rho_{0, \mathrm{alg}} \cdot \nabla \psi_{0}^{\mathbf{a}}\right\|_{\omega_{0}^{\mathbf{a}}}\right\}^{2},
$$

again as in (11.1a). Thus the efficiency of $\eta_{6.3}^{p}$ follows as in the proof of Theorem 11.1.

Efficiency of $\eta_{7.1}^{p}$ : Note that, using the minimization property (6.14),

$$
\left\|\sigma_{j, 6,3, \text { alg }}^{\mathbf{a}, \mathbf{a}^{\prime}}\right\|_{\omega^{\mathbf{a}, \mathbf{a}^{\prime}}} \leq\left\|\boldsymbol{\sigma}_{j, 7.1, \mathrm{alg}}^{\mathbf{a}, \mathbf{a}^{\prime}}\right\|_{\omega^{\mathbf{a}, \mathbf{a}^{\prime}}}
$$

However, using the constructive proofs in [16, Secs. 6.3 and 7.2] relying on minimizations in each single element of the form (7.2), we also get

$$
\left\|\sigma_{j, 7.1, \mathrm{alg}}^{\mathbf{a}, \mathbf{a}^{\prime}}\right\|_{\omega^{\mathbf{a}, \mathbf{a}^{\prime}}} \leq C_{\mathrm{st}}\left\|\sigma_{j, 6.3, \mathrm{alg}}^{\mathbf{a}, \mathbf{a}^{\prime}}\right\|_{\omega^{\mathbf{a}, \mathbf{a}^{\prime}}}
$$

where the constant $C_{\text {st }}$ of [16, Theorems 2.3 and 2.5] only depends on the shape regularity of the elements in the patch $\omega^{\mathbf{a}, \mathbf{a}^{\prime}}$. Thus, we conclude by the efficiency of $\eta_{6.3}^{p}$ established above.

Finally, we prove the efficiency of the upper bound based on the lowest-degree algebraic residual lifting of Sec. 8.

Theorem 11.3 (Efficiency of the upper bound $\eta_{\mathrm{alg}}^{0}$ on the algebraic error). Let $\eta_{\mathrm{alg}}^{0}$ be given by (10.11). There holds

$$
\eta_{\text {alg }}^{0} \leq \bar{C}_{\mathrm{alg}}^{\mathrm{eff}, 0}\left\|\mathbf{A}^{1 / 2} \nabla\left(u_{h}^{\mathrm{ex}}-u_{h}\right)\right\|
$$

where $\bar{C}_{\mathrm{alg}}^{\mathrm{eff}, 0}$ is a generic constant only depending on the shape regularity of the mesh $\mathscr{T}_{h}$, the space dimension $d$, the polynomial degree $p$, the number of mesh levels $J$, and the ratio of the largest to the smallest eigenvalue of the diffusion coefficient $\mathbf{A}$.

Proof. We again only reason for $\mathbf{A}=$ Id. First, we bound the norm of the local contributions $\left\|\boldsymbol{\sigma}_{j, 0, \text { alg }}^{\mathbf{a}}\right\| \|_{\omega_{j-1}^{\mathrm{a}}}$ by $\left\|\Pi_{j}^{0} r_{h}\right\|_{\omega_{j-1}^{\mathrm{a}}}$ and $\left\|\nabla \rho_{0, \mathrm{alg}}\right\|_{\omega_{j-1}^{\mathrm{a}}}$ as in the proof of Theorem 11.2 and in Step 2 of the proof of Theorem 11.1. Then we use $\left\|\Pi_{j}^{0} r_{h}\right\|_{K} \leq\left\|r_{h}\right\|_{K}$ (since $\Pi_{j}^{0}$ is a projection) and $\left\|r_{h}-\Pi_{J}^{0} r_{h}\right\|_{K} \leq\left\|r_{h}\right\|_{K}$. Finally, the proof follows using the Steps 3-6 of the proof of Theorem 11.1 above.

\section{Numerical results}

For the numerical experiments, we consider the same test problems and setting as in [28], so that we can asses the quality of our inexpensive bounds with respect to the original one in [28]. We consider the model problem (10.1) with $\mathbf{A}=\mathrm{Id}$ and three different choices of the domain $\Omega \in \mathbb{R}^{2}$ and of the exact solution $u$ :

$$
\begin{array}{lr}
\Omega:=(-1,1)^{2}, u(x, y):=\sin (2 \pi x) \sin (2 \pi y) & \text { sinus, } \\
\Omega:=(0,1)^{2}, u(x, y):=x(x-1) y(y-1) \exp \left(-100\left(\left(x-\frac{1}{2}\right)^{2}-\left(y-\frac{117}{1000}\right)^{2}\right)\right) & \text { peak, } \\
\Omega:=(-1,1)^{2} \backslash[0,1] \times[-1,0], u(r, \theta):=r^{2 / 3} \sin (2 \theta / 3) & \text { L-shape. }
\end{array}
$$


In the last case, we impose an inhomogeneous Dirichlet boundary condition corresponding to the prescribed exact solution. We consider the finite element method (10.2) with the polynomial degrees $p=1, \ldots, 4$. For each test problem, we start from an initial Delaunay triangulation of the domain $\Omega$ and consider four uniform refinement steps, so that $J=4$. We give in the tables and figures below the effectivity index defined as the ratio of the estimate (error upper bound) to the error,

$$
\text { eff. index }:=\frac{\text { estimate }}{\text { error }} \text {. }
$$

\subsection{Algebraic error bounds based on the $p$-degree polynomial lifting}

First, we present the results for bounds from Theorem 10.1 based on the liftings constructed in $p$-degree RTN space $\mathbf{V}_{J}^{p}$ in Sections 5-7. We present the results in the same way as in [28], to allow for a comparison. As an algebraic solver, we first consider a geometric multigrid method (MG) with V(5,0)-cycles, i.e., employing 5 Gauss-Seidel pre-smoothing iterations and no post-smoothing. Second, we consider a full multigrid (FMG) method using a single V(3,3)-cycle on each level (i.e. with 3 Gauss-Seidel preand post-smoothing iterations). Finally, we also examine the preconditioned conjugate gradient method (PCG) with an incomplete Cholesky preconditioner with the relative drop-off tolerance $10^{-4}$ (see, e.g., [33, Sec. 10.4]). Our starting iterate is the zero vector, and in the MG and PCG setting, we iterate until the algebraic error drops below $10 \%$ of the discretization error.

As presented in [28, Theorem 7.1], there also exists an upper bound on the total error $\left\|\nabla\left(u-u_{h}\right)\right\|$ in the form

$$
\left\|\nabla\left(u-u_{h}\right)\right\| \leq \eta_{\mathrm{tot}}^{p}:=\widetilde{\eta}_{\mathrm{dis}}+\eta_{\mathrm{osc}}+\eta_{\mathrm{alg}}^{p},
$$

where $\eta_{\mathrm{alg}}^{p}$ is given by (10.9). The term $\eta_{\text {osc }}$ measures the oscillation in the data $f$ and $\widetilde{\eta}_{\text {dis }}$ can be used to approximate the discretization error $\left\|\nabla\left(u-u_{h}^{\mathrm{ex}}\right)\right\|$. These two terms are independent of the construction

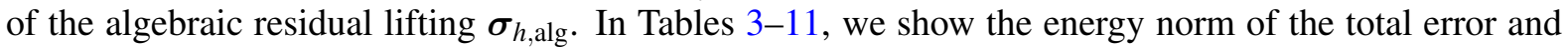
the efficiency of the upper bound determined by using in (12.1) the upper bounds on the algebraic error $\eta_{\mathrm{alg}}^{p}=\eta_{5.1}^{p}, \eta_{6.3}^{p}$, or $\eta_{7.1}^{p}$.

\begin{tabular}{lc|cccc|cccc|c} 
& MG & algebraic & \multicolumn{2}{c|}{ eff. index } & $\eta_{\mathrm{alg}}^{p}$ & total & \multicolumn{2}{c}{ eff. index } & $\eta_{\mathrm{tot}}^{p}$ & discretization \\
$p\left(N_{h}\right)$ & iter & error & $\eta_{5.1}^{p}$ & $\eta_{6.3}^{p}$ & $\eta_{7.1}^{p}$ & error & $\eta_{5.1}^{p}$ & $\eta_{6.3}^{p}$ & $\eta_{7.1}^{p}$ & error \\
\hline $1\left(3.5 \times 10^{4}\right)$ & 1 & 1.2 & 1.11 & 1.11 & 1.20 & 1.3 & 1.46 & 1.47 & 1.55 & $2.4 \times 10^{-1}$ \\
& 2 & $8.0 \times 10^{-2}$ & 1.13 & 1.16 & 1.27 & $2.5 \times 10^{-1}$ & 1.35 & 1.36 & 1.40 & \\
& 3 & $5.1 \times 10^{-3}$ & 1.15 & 1.17 & 1.30 & $2.4 \times 10^{-1}$ & 1.06 & 1.06 & 1.07 & \\
\hline $2\left(1.4 \times 10^{5}\right)$ & 1 & 1.2 & 1.10 & 1.11 & 1.39 & 1.2 & 1.48 & 1.50 & 1.77 & $2.9 \times 10^{-3}$ \\
& 2 & $1.1 \times 10^{-1}$ & 1.18 & 1.20 & 1.61 & $1.1 \times 10^{-1}$ & 1.77 & 1.79 & 2.20 & \\
& 3 & $2.8 \times 10^{-3}$ & 1.18 & 1.20 & 1.63 & $4.1 \times 10^{-3}$ & 1.66 & 1.67 & 1.97 & \\
& 4 & $9.6 \times 10^{-5}$ & 1.20 & 1.25 & 1.65 & $2.9 \times 10^{-3}$ & 1.05 & 1.05 & 1.06 & \\
\hline $3\left(3.2 \times 10^{5}\right)$ & 1 & $5.9 \times 10^{-1}$ & 1.09 & 1.17 & 1.42 & $5.9 \times 10^{-1}$ & 1.33 & 1.41 & 1.66 & $2.2 \times 10^{-5}$ \\
& 3 & $2.2 \times 10^{-3}$ & 1.19 & 1.18 & 1.59 & $2.2 \times 10^{-3}$ & 1.75 & 1.74 & 2.15 & \\
& 5 & $1.0 \times 10^{-5}$ & 1.19 & 1.18 & 1.60 & $2.4 \times 10^{-5}$ & 1.44 & 1.44 & 1.61 & \\
& 6 & $1.4 \times 10^{-6}$ & 1.18 & 1.18 & 1.60 & $2.2 \times 10^{-5}$ & 1.08 & 1.08 & 1.10 & \\
\hline $4\left(5.6 \times 10^{5}\right)$ & 1 & $4.5 \times 10^{-1}$ & 1.08 & 1.19 & 1.57 & $4.5 \times 10^{-1}$ & 1.39 & 1.49 & 1.94 & $1.5 \times 10^{-7}$ \\
& 4 & $1.2 \times 10^{-4}$ & 1.13 & 1.14 & 1.47 & $1.2 \times 10^{-4}$ & 1.58 & 1.59 & 1.92 & \\
& 7 & $6.4 \times 10^{-7}$ & 1.11 & 1.12 & 1.41 & $6.5 \times 10^{-7}$ & 1.53 & 1.54 & 1.82 & \\
& 10 & $8.3 \times 10^{-9}$ & 1.12 & 1.12 & 1.41 & $1.5 \times 10^{-7}$ & 1.06 & 1.06 & 1.08 &
\end{tabular}

Table 3: Sinus problem, multigrid V-cycles: efficiency of the error upper bounds of Theorem 10.1 


\begin{tabular}{|c|c|c|c|c|c|c|c|c|c|}
\hline & $\begin{array}{l}\text { algebraic } \\
\text { error }\end{array}$ & & $p$ & & $\begin{array}{l}\text { total } \\
\text { error }\end{array}$ & & & & error \\
\hline $\left.0^{4}\right)$ & $07 \times 1-4$ & 1.03 & $\begin{array}{l}1.3 \\
1.07\end{array}$ & 1.20 & $4 \times 10^{-1}$ & $\begin{array}{l}1.1 \\
.04\end{array}$ & $\begin{array}{l}1.30 \\
1.04\end{array}$ & 1.04 & $2.4 \times 10^{-1}$ \\
\hline & & 10 & 1.18 & 1.48 & & 1.02 & 1.02 & 1.02 & \\
\hline & & 1.08 & 1.06 & .55 & $2.2\rangle$ & 1.12 & 1.1 & 1.18 & \\
\hline $4\left(5.6 \times 10^{5}\right)$ & $1 \times 10^{-8}$ & 1.03 & 1.10 & 1.35 & $1.6 \times 10^{-7}$ & 1.29 & 1.31 & 1.39 & $1.5 \times 10^{-7}$ \\
\hline
\end{tabular}

Table 4: Sinus problem, one full multigrid cycle: efficiency of the error upper bounds of Theorem 10.1

\begin{tabular}{|c|c|c|c|c|c|c|c|c|c|c|}
\hline$p\left(N_{h}\right)$ & $\begin{array}{l}\text { PCG } \\
\text { iter }\end{array}$ & $\begin{array}{c}\text { algebraic } \\
\text { error }\end{array}$ & $\begin{array}{c}\text { eff } \\
\eta_{5.1}^{p}\end{array}$ & $\begin{array}{l}\text { index } \\
\eta_{6.3}^{p}\end{array}$ & $\begin{array}{l}\eta_{\text {alg }}^{\rho} \\
\eta_{7.1}^{p}\end{array}$ & $\begin{array}{l}\text { total } \\
\text { error }\end{array}$ & & $\begin{array}{c}\text { index } \\
\eta_{6.3}^{p}\end{array}$ & & $\begin{array}{c}\text { discretization } \\
\text { error }\end{array}$ \\
\hline \multirow[t]{2}{*}{$1\left(3.5 \times 10^{4}\right)$} & 3 & $6.4 \times 10^{-2}$ & 1.01 & 1.01 & 1.15 & $2.5 \times 10^{-1}$ & 1.26 & 1.26 & 1.30 & \multirow[t]{2}{*}{$2.4 \times 10^{-1}$} \\
\hline & 6 & $1.3 \times 10^{-2}$ & 1.01 & 1.01 & 1.16 & $2.4 \times 10^{-1}$ & 1.09 & 1.09 & 1.10 & \\
\hline \multirow[t]{3}{*}{$2\left(1.4 \times 10^{5}\right)$} & 5 & $5.2 \times 10^{-2}$ & 1.01 & 1.02 & 1.17 & $5.2 \times 10^{-2}$ & 1.09 & 1.11 & 1.25 & \multirow[t]{3}{*}{$2.9 \times 10^{-3}$} \\
\hline & 10 & $5.6 \times 10^{-3}$ & 1.00 & 1.01 & 1.15 & $6.3 \times 10^{-3}$ & 1.36 & 1.37 & 1.49 & \\
\hline & 15 & $1.2 \times 10^{-4}$ & 1.01 & 1.03 & 1.19 & $2.9 \times 10^{-3}$ & 1.05 & 1.05 & 1.06 & \\
\hline \multirow[t]{4}{*}{$3\left(3.2 \times 10^{5}\right)$} & 11 & $4.2 \times 10^{-2}$ & 1.00 & 1.03 & 1.17 & $4.2 \times 10^{-2}$ & 1.05 & 1.07 & 1.22 & \multirow[t]{4}{*}{$2.2 \times 10^{-5}$} \\
\hline & 22 & $6.3 \times 10^{-3}$ & 1.00 & 1.03 & 1.17 & $6.3 \times 10^{-3}$ & 1.05 & 1.08 & 1.22 & \\
\hline & 33 & $6.7 \times 10^{-5}$ & 1.01 & 1.06 & 1.20 & $7.0 \times 10^{-5}$ & 1.29 & 1.34 & 1.47 & \\
\hline & 44 & $8.0 \times 10^{-7}$ & 1.00 & 1.03 & 1.17 & $2.2 \times 10^{-5}$ & 1.04 & 1.04 & 1.05 & \\
\hline \multirow[t]{4}{*}{$4\left(5.6 \times 10^{5}\right)$} & 15 & $2.6 \times 10^{-2}$ & 1.00 & 1.01 & 1.15 & $2.6 \times 10^{-2}$ & 1.05 & 1.06 & 1.20 & \multirow[t]{4}{*}{$1.5 \times 10^{-7}$} \\
\hline & 30 & $7.0 \times 10^{-4}$ & 1.01 & 1.03 & 1.17 & $7.0 \times 10^{-4}$ & 1.08 & 1.10 & 1.24 & \\
\hline & 45 & $9.1 \times 10^{-7}$ & 1.00 & 1.02 & 1.16 & $9.3 \times 10^{-7}$ & 1.16 & 1.18 & 1.32 & \\
\hline & 60 & $2.5 \times 10^{-9}$ & 1.01 & 1.04 & 1.19 & $1.5 \times 10^{-7}$ & 1.02 & 1.02 & 1.02 & \\
\hline
\end{tabular}

Table 5: Sinus problem, PCG iterations: efficiency of the error upper bounds of Theorem 10.1

\begin{tabular}{lc|cccc|cccc|c} 
& MG & algebraic & \multicolumn{2}{c|}{ eff. index } & $\eta_{\text {alg }}^{p}$ & \multicolumn{2}{c}{ total } & \multicolumn{3}{c}{ eff. index } \\
$p\left(N_{h}\right)$ & iter & error & $\eta_{5.1}^{p}$ & $\eta_{6.3}^{p}$ & $\eta_{7.1}^{p}$ & error & $\eta_{5.1}^{p}$ & $\eta_{6.3}^{p}$ & $\eta_{7.1}^{p}$ & error \\
\hline $1\left(9.3 \times 10^{3}\right)$ & 1 & $6.1 \times 10^{-3}$ & 1.13 & 1.13 & 1.23 & $6.9 \times 10^{-3}$ & 1.61 & 1.61 & 1.70 & $3.3 \times 10^{-3}$ \\
& 2 & $1.9 \times 10^{-4}$ & 1.13 & 1.15 & 1.27 & $3.3 \times 10^{-3}$ & 1.10 & 1.10 & 1.11 & \\
\hline $2\left(3.8 \times 10^{4}\right)$ & 1 & $7.5 \times 10^{-3}$ & 1.13 & 1.14 & 1.50 & $7.5 \times 10^{-3}$ & 1.61 & 1.62 & 1.98 & $1.1 \times 10^{-4}$ \\
& 2 & $4.5 \times 10^{-4}$ & 1.17 & 1.18 & 1.59 & $4.6 \times 10^{-4}$ & 1.76 & 1.77 & 2.17 & \\
& 3 & $8.1 \times 10^{-6}$ & 1.17 & 1.18 & 1.61 & $1.1 \times 10^{-4}$ & 1.10 & 1.10 & 1.13 & \\
\hline $3\left(8.5 \times 10^{4}\right)$ & 1 & $4.9 \times 10^{-3}$ & 1.10 & 1.17 & 1.44 & $4.9 \times 10^{-3}$ & 1.40 & 1.47 & 1.73 & $2.9 \times 10^{-6}$ \\
& 3 & $1.3 \times 10^{-5}$ & 1.18 & 1.18 & 1.59 & $1.3 \times 10^{-5}$ & 1.75 & 1.74 & 2.15 & \\
& 5 & $7.8 \times 10^{-9}$ & 1.17 & 1.16 & 1.64 & $2.9 \times 10^{-6}$ & 1.01 & 1.01 & 1.01 & \\
\hline $4\left(1.5 \times 10^{5}\right)$ & 1 & $4.4 \times 10^{-3}$ & 1.09 & 1.21 & 1.69 & $4.4 \times 10^{-3}$ & 1.44 & 1.56 & 2.04 & $6.3 \times 10^{-8}$ \\
& 3 & $1.8 \times 10^{-5}$ & 1.15 & 1.16 & 1.47 & $1.8 \times 10^{-5}$ & 1.67 & 1.68 & 1.99 & \\
& 5 & $2.4 \times 10^{-8}$ & 1.11 & 1.12 & 1.41 & $6.8 \times 10^{-8}$ & 1.34 & 1.34 & 1.44 & \\
& 6 & $1.1 \times 10^{-9}$ & 1.11 & 1.11 & 1.37 & $6.3 \times 10^{-8}$ & 1.02 & 1.02 & 1.03 &
\end{tabular}

Table 6: Peak problem, multigrid V-cycles: efficiency of the error upper bounds of Theorem 10.1

\begin{tabular}{l|cccc|cccc|c} 
& algebraic & \multicolumn{3}{|c|}{ eff. index } & $\eta_{\text {alg }}^{p}$ & total & \multicolumn{2}{c|}{ eff. index $\eta_{\text {tot }}^{p}$} & discretization \\
$p\left(N_{h}\right)$ & error & $\eta_{5.1}^{p}$ & $\eta_{6.3}^{p}$ & $\eta_{7.1}^{p}$ & error & $\eta_{5.1}^{p}$ & $\eta_{6.3}^{p}$ & $\eta_{7.1}^{p}$ & error \\
\hline $1\left(9.3 \times 10^{3}\right)$ & $1.8 \times 10^{-5}$ & 1.02 & 1.09 & 1.27 & $3.3 \times 10^{-3}$ & 1.04 & 1.04 & 1.05 & $3.3 \times 10^{-3}$ \\
\hline $2\left(3.8 \times 10^{4}\right)$ & $1.9 \times 10^{-7}$ & 1.07 & 1.10 & 1.33 & $1.1 \times 10^{-4}$ & 1.01 & 1.01 & 1.01 & $1.1 \times 10^{-4}$ \\
\hline $3\left(8.5 \times 10^{4}\right)$ & $2.2 \times 10^{-7}$ & 1.08 & 1.06 & 1.56 & $2.9 \times 10^{-6}$ & 1.08 & 1.08 & 1.12 & $2.9 \times 10^{-6}$ \\
\hline $4\left(1.5 \times 10^{5}\right)$ & $9.1 \times 10^{-9}$ & 1.05 & 1.10 & 1.40 & $6.4 \times 10^{-8}$ & 1.14 & 1.15 & 1.19 & $6.3 \times 10^{-8}$
\end{tabular}

Table 7: Peak problem, one full multigrid cycle: efficiency of the error upper bounds of Theorem 10.1 


\begin{tabular}{lc|cccc|cccc|c} 
& PCG & algebraic & \multicolumn{2}{c|}{ eff. index } & $\eta_{\text {alg }}^{p}$ & total & \multicolumn{2}{c}{ eff. index $\eta_{\text {tot }}^{p}$} & discretization \\
$p\left(N_{h}\right)$ & iter & error & $\eta_{5.1}^{p}$ & $\eta_{6.3}^{p}$ & $\eta_{7.1}^{p}$ & error & $\eta_{5.1}^{p}$ & $\eta_{6.3}^{p}$ & $\eta_{7.1}^{p}$ & error \\
\hline $1\left(9.3 \times 10^{3}\right)$ & 2 & $1.0 \times 10^{-3}$ & 1.01 & 1.02 & 1.17 & $3.5 \times 10^{-3}$ & 1.29 & 1.29 & 1.34 & $3.3 \times 10^{-3}$ \\
& 4 & $9.1 \times 10^{-5}$ & 1.02 & 1.05 & 1.17 & $3.3 \times 10^{-3}$ & 1.07 & 1.07 & 1.07 & \\
\hline $2\left(3.8 \times 10^{4}\right)$ & 4 & $6.1 \times 10^{-4}$ & 1.01 & 1.02 & 1.18 & $6.2 \times 10^{-4}$ & 1.20 & 1.21 & 1.37 & $1.1 \times 10^{-4}$ \\
& 8 & $3.2 \times 10^{-6}$ & 1.01 & 1.06 & 1.21 & $1.1 \times 10^{-4}$ & 1.04 & 1.04 & 1.05 & \\
\hline $3\left(8.5 \times 10^{4}\right)$ & 7 & $1.1 \times 10^{-3}$ & 1.00 & 1.02 & 1.19 & $1.1 \times 10^{-3}$ & 1.04 & 1.05 & 1.22 & $2.9 \times 10^{-6}$ \\
& 14 & $2.2 \times 10^{-5}$ & 1.02 & 1.08 & 1.23 & $2.2 \times 10^{-5}$ & 1.22 & 1.28 & 1.42 & \\
& 21 & $4.8 \times 10^{-8}$ & 1.01 & 1.06 & 1.21 & $2.9 \times 10^{-6}$ & 1.02 & 1.02 & 1.03 & \\
\hline $4\left(1.5 \times 10^{5}\right)$ & 7 & $1.1 \times 10^{-3}$ & 1.00 & 1.02 & 1.18 & $1.1 \times 10^{-3}$ & 1.06 & 1.07 & 1.23 & $6.3 \times 10^{-8}$ \\
& 14 & $4.9 \times 10^{-5}$ & 1.01 & 1.07 & 1.22 & $4.9 \times 10^{-5}$ & 1.11 & 1.17 & 1.32 & \\
& 21 & $2.0 \times 10^{-7}$ & 1.01 & 1.07 & 1.19 & $2.1 \times 10^{-7}$ & 1.28 & 1.34 & 1.46 & \\
& 28 & $1.9 \times 10^{-10}$ & 1.01 & 1.07 & 1.23 & $6.3 \times 10^{-8}$ & 1.01 & 1.01 & 1.01 &
\end{tabular}

Table 8: Peak problem, PCG iterations: efficiency of the error upper bounds of Theorem 10.1

\begin{tabular}{lc|cccc|cccc|c} 
& MG & algebraic & \multicolumn{2}{c|}{ eff. index } & $\eta_{\text {alg }}^{p}$ & total & \multicolumn{3}{c}{ eff. index $\eta_{\text {tot }}^{p}$} & discretization \\
$p\left(N_{h}\right)$ & iter & error & $\eta_{5.1}^{p}$ & $\eta_{6.3}^{p}$ & $\eta_{7.1}^{p}$ & error & $\eta_{5.1}^{p}$ & $\eta_{6.3}^{p}$ & $\eta_{7.1}^{p}$ & error \\
\hline $1\left(2.5 \times 10^{4}\right)$ & 1 & 1.4 & 1.14 & 1.18 & 1.37 & 1.4 & 1.60 & 1.64 & 1.83 & $2.2 \times 10^{-2}$ \\
& 2 & $6.7 \times 10^{-2}$ & 1.14 & 1.19 & 1.38 & $7.0 \times 10^{-2}$ & 1.61 & 1.65 & 1.84 & \\
& 3 & $4.3 \times 10^{-3}$ & 1.16 & 1.25 & 1.59 & $2.3 \times 10^{-2}$ & 1.37 & 1.39 & 1.45 & \\
& 4 & $4.1 \times 10^{-4}$ & 1.17 & 1.31 & 1.76 & $2.2 \times 10^{-2}$ & 1.22 & 1.22 & 1.23 & \\
\hline $2\left(1.0 \times 10^{5}\right)$ & 1 & 2.6 & 1.19 & 1.22 & 1.74 & 2.6 & 1.78 & 1.81 & 2.33 & $8.9 \times 10^{-3}$ \\
& 2 & $8.9 \times 10^{-2}$ & 1.19 & 1.20 & 1.64 & $8.9 \times 10^{-2}$ & 1.79 & 1.80 & 2.24 & \\
& 3 & $2.2 \times 10^{-3}$ & 1.18 & 1.21 & 1.64 & $9.2 \times 10^{-3}$ & 1.55 & 1.56 & 1.66 & \\
& 4 & $8.6 \times 10^{-5}$ & 1.19 & 1.25 & 1.70 & $8.9 \times 10^{-3}$ & 1.32 & 1.32 & 1.32 & \\
\hline $3\left(2.3 \times 10^{5}\right)$ & 1 & 2.4 & 1.19 & 1.20 & 1.59 & 2.4 & 1.72 & 1.74 & 2.12 & $5.3 \times 10^{-3}$ \\
& 2 & $1.1 \times 10^{-1}$ & 1.20 & 1.19 & 1.59 & $1.1 \times 10^{-1}$ & 1.76 & 1.76 & 2.16 & \\
& 3 & $3.6 \times 10^{-3}$ & 1.18 & 1.17 & 1.61 & $6.4 \times 10^{-3}$ & 1.89 & 1.88 & 2.13 & \\
& 4 & $1.8 \times 10^{-4}$ & 1.17 & 1.16 & 1.66 & $5.3 \times 10^{-3}$ & 1.48 & 1.48 & 1.49 & \\
\hline $4\left(4.0 \times 10^{5}\right)$ & 1 & 2.6 & 1.18 & 1.25 & 1.61 & 2.6 & 1.68 & 1.75 & 2.11 & $3.8 \times 10^{-3}$ \\
& 2 & $1.3 \times 10^{-1}$ & 1.18 & 1.18 & 1.50 & $1.3 \times 10^{-1}$ & 1.71 & 1.72 & 2.03 & \\
& 3 & $6.0 \times 10^{-3}$ & 1.16 & 1.15 & 1.46 & $7.1 \times 10^{-3}$ & 1.87 & 1.87 & 2.12 & \\
& 4 & $3.5 \times 10^{-4}$ & 1.13 & 1.13 & 1.44 & $3.8 \times 10^{-3}$ & 1.57 & 1.57 & 1.60 &
\end{tabular}

Table 9: L-shape problem, multigrid V-cycles: efficiency of the error upper bounds of Theorem 10.1

\begin{tabular}{l|cccc|cccc|c} 
& algebraic & \multicolumn{2}{|c|}{ eff. index } & $\eta_{\text {alg }}^{p}$ & total & \multicolumn{2}{c|}{ eff. index $\eta_{\text {tot }}^{p}$} & discretization \\
$p\left(N_{h}\right)$ & error & $\eta_{5.1}^{p}$ & $\eta_{6.3}^{p}$ & $\eta_{7.1}^{p}$ & error & $\eta_{5.1}^{p}$ & $\eta_{6.3}^{p}$ & $\eta_{7.1}^{p}$ & error \\
\hline $1\left(2.5 \times 10^{4}\right)$ & $4.4 \times 10^{-4}$ & 1.11 & 1.27 & 1.79 & $2.2 \times 10^{-2}$ & 1.22 & 1.22 & 1.23 & $2.2 \times 10^{-2}$ \\
\hline $2\left(1.0 \times 10^{5}\right)$ & $8.0 \times 10^{-5}$ & 1.12 & 1.44 & 2.04 & $8.9 \times 10^{-3}$ & 1.32 & 1.32 & 1.33 & $8.9 \times 10^{-3}$ \\
\hline $3\left(2.3 \times 10^{5}\right)$ & $5.5 \times 10^{-5}$ & 1.09 & 1.21 & 1.84 & $5.3 \times 10^{-3}$ & 1.45 & 1.45 & 1.46 & $5.3 \times 10^{-3}$ \\
\hline $4\left(4.0 \times 10^{5}\right)$ & $7.2 \times 10^{-5}$ & 1.08 & 1.24 & 1.72 & $3.8 \times 10^{-3}$ & 1.49 & 1.49 & 1.50 & $3.8 \times 10^{-3}$
\end{tabular}

Table 10: L-shape problem, one full multigrid cycle: efficiency of the error upper bounds of Theorem 10.1 


\begin{tabular}{|c|c|c|c|c|c|c|c|c|c|c|}
\hline \multirow[b]{2}{*}{$p\left(N_{h}\right)$} & \multirow{2}{*}{$\begin{array}{l}\text { PCG } \\
\text { iter }\end{array}$} & \multirow{2}{*}{$\begin{array}{c}\text { algebraic } \\
\text { error }\end{array}$} & \multicolumn{3}{|c|}{ eff. index $\eta_{\mathrm{alg}}^{p}$} & \multirow{2}{*}{$\begin{array}{l}\text { total } \\
\text { error }\end{array}$} & \multicolumn{3}{|c|}{ eff. index $\eta_{\text {tot }}^{p}$} & \multirow{2}{*}{$\begin{array}{c}\text { discretization } \\
\text { error }\end{array}$} \\
\hline & & & $\eta_{5.1}^{p}$ & $\eta_{6.3}^{p}$ & $\eta_{7.1}^{p}$ & & $\eta_{5,1}^{p}$ & $\eta_{6.3}^{p}$ & $\eta_{7.1}^{p}$ & \\
\hline \multirow[t]{2}{*}{$1\left(2.5 \times 10^{4}\right)$} & 4 & $8.9 \times 10^{-2}$ & 1.02 & 1.05 & 1.18 & $9.1 \times 10^{-2}$ & 1.26 & 1.29 & 1.42 & \multirow[t]{2}{*}{$2.2 \times 10^{-2}$} \\
\hline & 8 & $3.8 \times 10^{-4}$ & 1.01 & 1.03 & 1.17 & $2.2 \times 10^{-2}$ & 1.22 & 1.22 & 1.22 & \\
\hline \multirow[t]{3}{*}{$2\left(1.0 \times 10^{5}\right)$} & 4 & $6.2 \times 10^{-1}$ & 1.01 & 1.03 & 1.18 & $6.2 \times 10^{-1}$ & 1.07 & 1.09 & 1.24 & \multirow[t]{3}{*}{$8.9 \times 10^{-3}$} \\
\hline & 8 & $6.0 \times 10^{-3}$ & 1.01 & 1.04 & 1.19 & $1.1 \times 10^{-2}$ & 1.65 & 1.67 & 1.75 & \\
\hline & 12 & $1.9 \times 10^{-4}$ & 1.01 & 1.03 & 1.18 & $8.9 \times 10^{-3}$ & 1.33 & 1.33 & 1.33 & \\
\hline \multirow[t]{4}{*}{$3\left(2.3 \times 10^{5}\right)$} & 7 & 1.0 & 1.00 & 1.03 & 1.17 & 1.0 & 1.05 & 1.07 & 1.22 & \multirow[t]{4}{*}{$5.3 \times 10^{-3}$} \\
\hline & 14 & $3.1 \times 10^{-2}$ & 1.01 & 1.04 & 1.19 & $3.1 \times 10^{-2}$ & 1.24 & 1.27 & 1.42 & \\
\hline & 21 & $1.7 \times 10^{-3}$ & 1.00 & 1.03 & 1.15 & $5.6 \times 10^{-3}$ & 1.68 & 1.69 & 1.72 & \\
\hline & 28 & $9.6 \times 10^{-5}$ & 1.00 & 1.03 & 1.18 & $5.3 \times 10^{-3}$ & 1.46 & 1.46 & 1.46 & \\
\hline \multirow[t]{4}{*}{$4\left(4.0 \times 10^{5}\right)$} & 7 & 1.2 & 1.01 & 1.02 & 1.17 & 1.2 & 1.08 & 1.10 & 1.25 & \multirow[t]{4}{*}{$3.8 \times 10^{-3}$} \\
\hline & 14 & $5.0 \times 10^{-2}$ & 1.01 & 1.04 & 1.18 & $5.1 \times 10^{-2}$ & 1.14 & 1.17 & 1.31 & \\
\hline & 21 & $3.4 \times 10^{-3}$ & 1.00 & 1.03 & 1.16 & $5.0 \times 10^{-3}$ & 1.77 & 1.78 & 1.87 & \\
\hline & 28 & $1.8 \times 10^{-4}$ & 1.01 & 1.04 & 1.18 & $3.8 \times 10^{-3}$ & 1.52 & 1.52 & 1.53 & \\
\hline
\end{tabular}

Table 11: L-shape problem, PCG iterations: efficiency of the error upper bounds of Theorem 10.1

The experiments confirm excellent efficiency of $\eta_{5.1}^{p}$ (reported already in [28, Sec. 9]) and also of the new upper bound $\eta_{6.3}^{p}$; typically, $\eta_{6.3}^{p}$ is very close to $\eta_{5.1}^{p}$. The effectivity index of $\eta_{7.1}^{p}$ is slightly worse but still, in most of the experiments and iterations, its value is below 2, which we consider very satisfactory. We also note that the efficiency of all the bounds based on the $p$-degree polynomial lifting deteriorates in the experiments neither with the increasing polynomial degree $p$ of the FEM approximation, nor with the number of levels $J$, though we could not prove these two properties rigorously in Sec. 11 .

\subsection{Algebraic error bound based on the approximate lowest-degree polynomial lifting}

We now examine the efficiency of the upper bound $\eta_{\mathrm{alg}}^{0}$ from Theorem 10.2, based on the algebraic residual lifting constructed in the lowest-degree RTN space $\mathbf{V}_{J}^{0}$ in Sec. 8. As remarked in Sec. 10.4, we expect a deterioration of the upper bound for increasing polynomial degree $p$ of the FEM approximation. Here, we consider iterations of V-cycle multigrid solver with 5 pre-smoothing Gauss-Seidel iterations and no postsmoothing. We still use $J=4$ levels and the sizes of the linear systems vary from $O\left(10^{4}\right)$ for $p=1$ to $O\left(10^{5}\right)$ for $p=4$.

The efficiency observed in Figures 7-9 is in particular satisfactory for $p=1$, where it stays close to 2 in the peak, respectively, to 2.5 in the sinus and L-shape test problems. One can note a significant deterioration of the efficiency between $p=1$ and $p=2$, while the deterioration seems less substantial between higher polynomial degrees. Nevertheless, the efficiency stays in all the numerical experiments on all iterations bounded by $p+2$. Taking into account the importantly reduced computational cost of constructing the lowest-degree polynomial approximate lifting of the algebraic residual, we believe that the guaranteed upper bound $\eta_{\mathrm{alg}}^{0}$ of Theorem 10.2 can be of interest in many applications.

\subsection{A timing comparison}

We finally present a timing comparison of the construction proposed in [28] with the present cheaper variants. For this purpose, we consider the peak problem and measure the time needed for one construction of the upper bounds $\eta_{5.1}^{p}, \eta_{6.3}^{p}, \eta_{7.1}^{p}$, and $\eta_{\mathrm{alg}}^{0}$. The constructions are implemented in MATLAB in a sequential code with an advanced use of build-in functions and vectorization. A mean value of ten runs is depicted in Figure 10, with times in seconds. The experiments were run on a laptop with Intel Core i5-8265U CPU and 8GB RAM. We trust the reader to understand the trickiness inherent to such implementation- and machine-dependent measurement. 


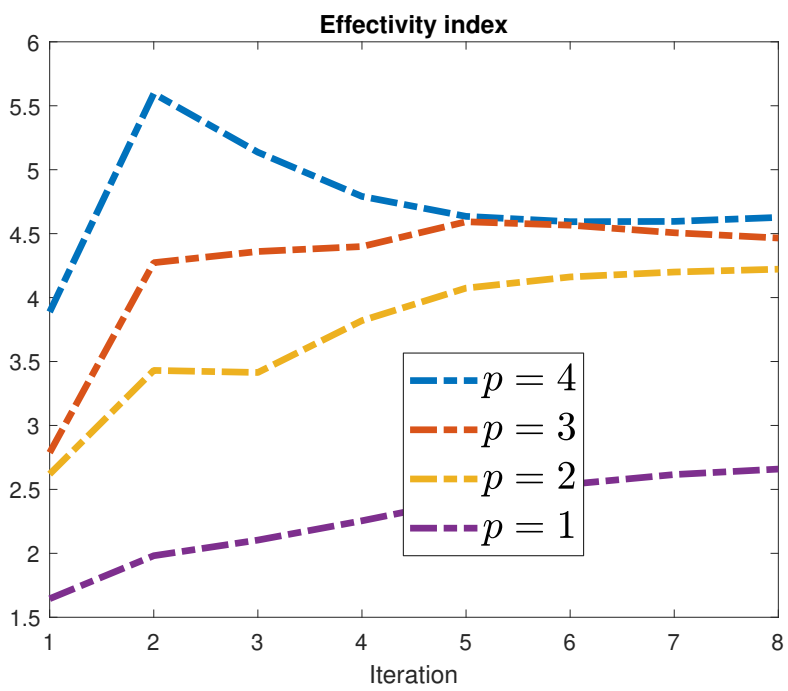

Figure 7: Sinus problem, multigrid V-cycles: efficiency of the bound $\eta_{\mathrm{alg}}^{0}$ of Theorem 10.2 for varying polynomial degree $p$ of FEM approximation

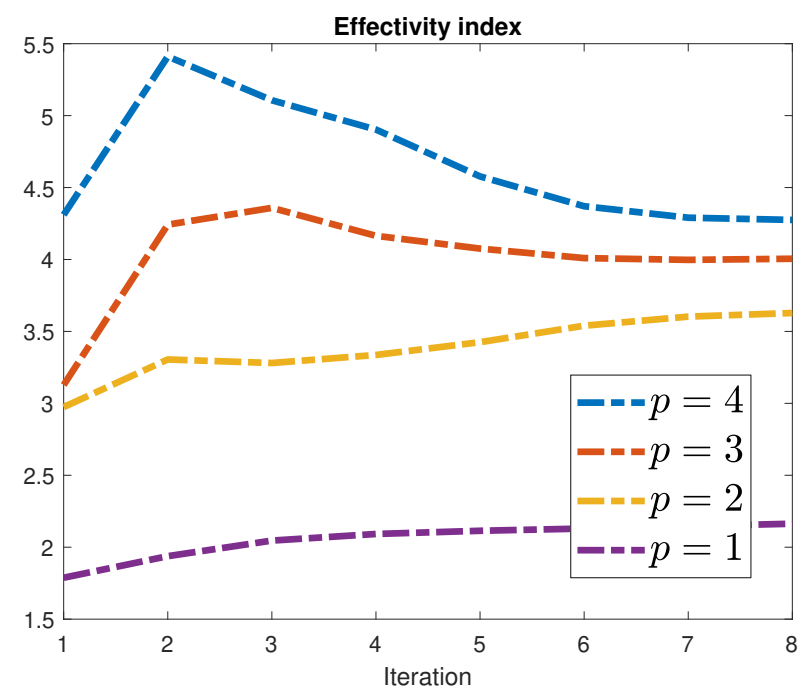

Figure 8: Peak problem, multigrid V-cycles: efficiency of the bound $\eta_{\mathrm{alg}}^{0}$ of Theorem 10.2 for varying polynomial degree $p$ of FEM approximation 


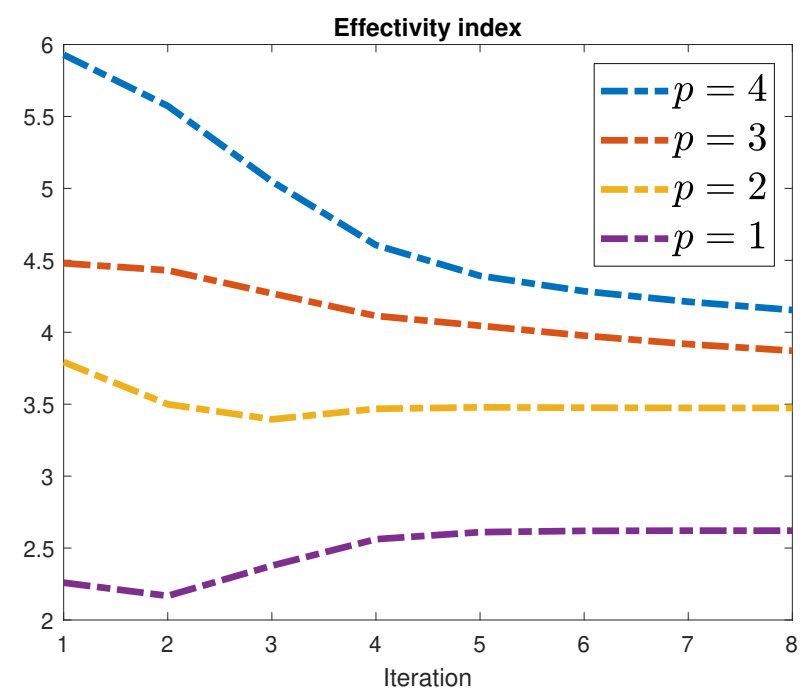

Figure 9: L-shape problem, multigrid V-cycles: efficiency of the bound $\eta_{\mathrm{alg}}^{0}$ of Theorem 10.2 for varying polynomial degree $p$ of FEM approximation

From Figure 10, one can see that the new bounds $\eta_{6.3}^{p}, \eta_{7.1}^{p}$, and $\eta_{\text {alg }}^{0}$ proposed in this paper offer a significant speed-up with respect to the original bound $\eta_{5.1}^{p}$ from [28]. As expected, the time to calculate the bound $\eta_{\mathrm{alg}}^{0}$ based on the lowest-degree polynomial approximate lifting depends on the polynomial degree only very mildly - the grow is caused by the fact that $r_{h}$ is $p$-order polynomial and the timing could be further reduced by implementing the quadrature rules in the construction more carefully.

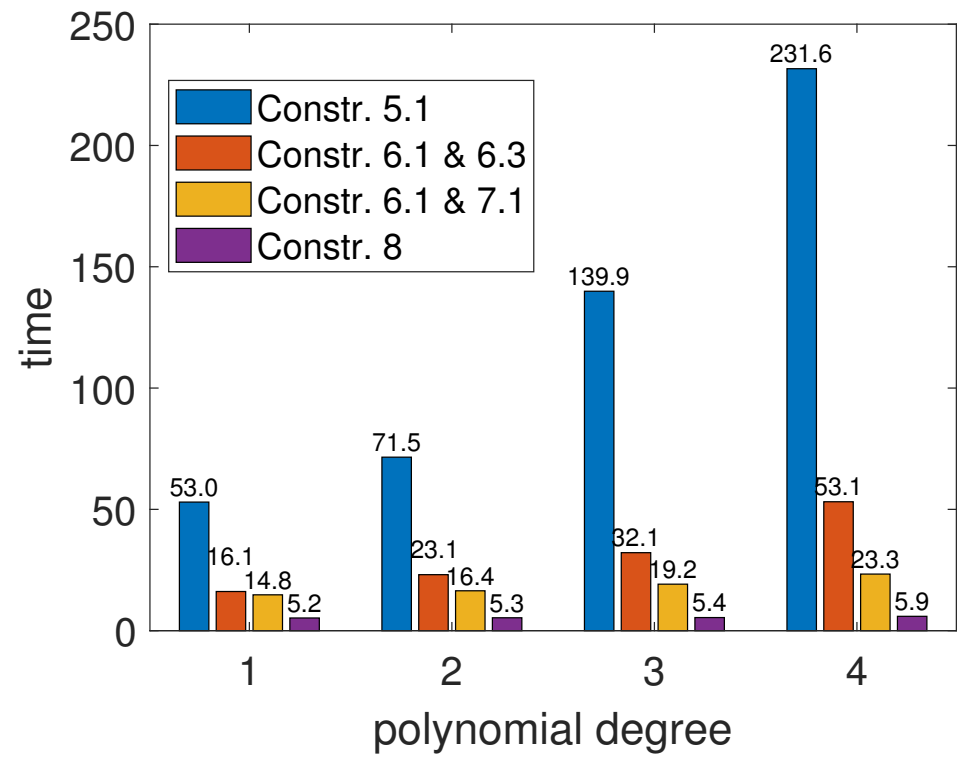

Figure 10: Timing comparison (in seconds) of the various constructions of Secs. 5-8 for varying polynomial degree in the peak problem.

We are well aware of the fact that the timings in Figure 10 are very high, which is partially because of the implementation (we use MATLAB in our codes) and because of the fact that no parallelization is used. In Figure 11, we therefore provide results of another experiment, still using MATLAB, where all the inter-level operations like (5.2) or (6.9) and the evaluations (10.9) and (10.11) are involved but on each level $1 \leq j \leq J$, we only perform a solve on one patch subdomain $\omega_{j-1}^{\mathrm{a}}$. This can be regarded as 
a lower bound on the timing when parallelization is used - recall that all the local problems on $\omega_{j-1}^{\mathbf{a}}$ in Secs. 5-8 are mutually independent and thus fully parallelizable. One can again see that the new constructions, which work with the high-order RTN functions only on the last level $j=J$ (in Secs. 5-7), or not at all (in Sec. 8) are importantly faster. Further speed-ups, in particular on at least partially structured meshes, are discussed in [28, Sec. 8].

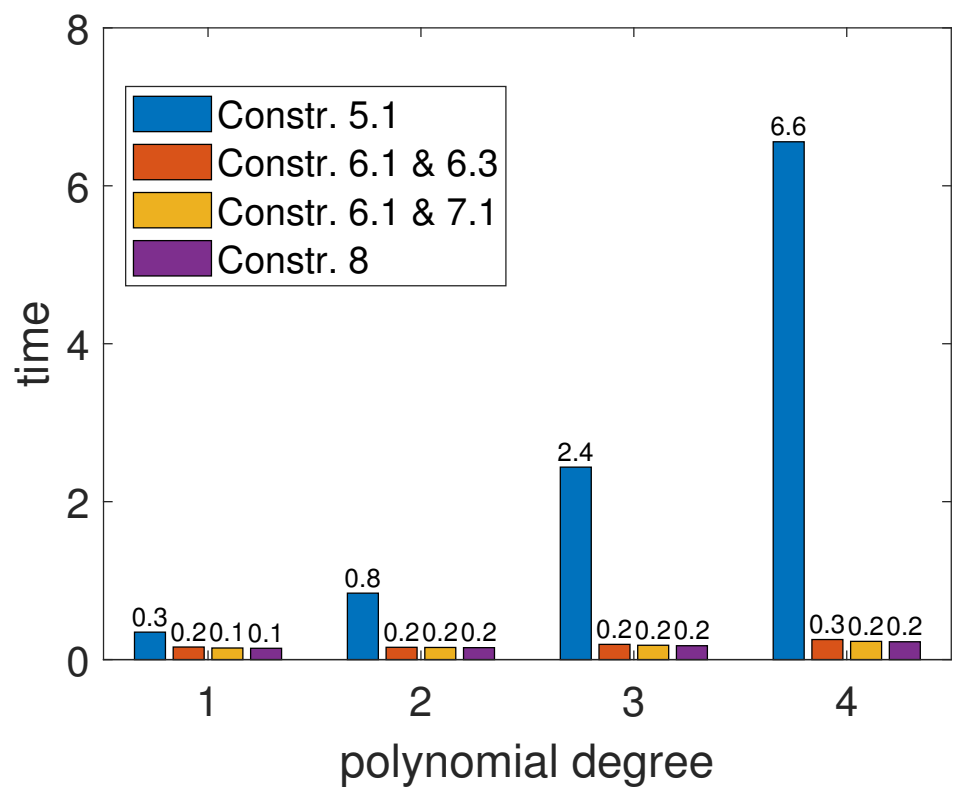

Figure 11: Timing comparison (in seconds) of the various constructions of Secs. 5-8 for varying polynomial degree in the peak problem. Here the timing involves all the inter-level operations but only a solve on one patch $\omega_{j-1}^{\mathrm{a}}$ on each level.

\section{Conclusions}

In this contribution, we presented new constructions of (approximate) liftings of a piecewise polynomial function. As showed in [28], this can be used to recover mass balance for any problem, any numerical discretization, and any situation. Here we rather used these liftings to construct guaranteed upper bounds on the algebraic error in arbitrary-order conforming discretization of the model diffusion problem. Together with the results of $[28,29]$, one can also monitor the total and discretizations errors.

The presented constructions of the liftings are significantly cheaper with respect to those of [28], while still preserving the same theoretical properties, i.e., guaranteed upper bound on the algebraic error and efficiency. The numerical experiments confirm the theoretical expectations and lead to very satisfactory effectivity indices of the estimators. Moreover, for the liftings of Sections 6-7, of the same polynomial order $p$ as that of the FEM discretization, we numerically observe $p$-robustness. This is not the case for the bounds based on the lowest-degree polynomial approximate lifting of Sec. 8, which attained in our numerical experiments effectivity indices below $p+2$. We, however, note that the lowestdegree polynomial approximate lifting has the computational cost further significantly reduced (allowing in addition to easily avoid any computer implementation of the Raviart-Thomas-Nédélec spaces, see Algorithm 4) and, therefore, it can be of interest in many applications. 


\section{References}

[1] M. Arioli, E. H. Georgoulis, and D. Loghin. Stopping criteria for adaptive finite element solvers. SIAM J. Sci. Comput., 35(3):A1537-A1559, 2013.

[2] M. Arioli, D. Loghin, and A. J. Wathen. Stopping criteria for iterations in finite element methods. Numer. Math., 99(3):381-410, 2005.

[3] D. N. Arnold, R. S. Falk, and R. Winther. Preconditioning in $H($ div $)$ and applications. Math. Comp., 66(219):957-984, 1997.

[4] D. N. Arnold, R. S. Falk, and R. Winther. Multigrid in $H$ (div) and H(curl). Numer. Math., 85(2):197-217, 2000.

[5] D. Bai and A. Brandt. Local mesh refinement multilevel techniques. SIAM J. Sci. Statist. Comput., 8(2):109-134, 1987.

[6] R. E. Bank and A. H. Sherman. An adaptive, multilevel method for elliptic boundary value problems. Computing, 26(2):91-105, 1981.

[7] R. E. Bank and R. K. Smith. A posteriori error estimates based on hierarchical bases. SIAM J. Numer. Anal., 30(4):921-935, 1993.

[8] R. Becker, C. Johnson, and R. Rannacher. Adaptive error control for multigrid finite element methods. Computing, 55(4):271-288, 1995.

[9] J. Blechta, J. Málek, and M. Vohralík. Localization of the $W^{-1, q}$ norm for local a posteriori efficiency. IMA J. Numer. Anal., 40(2):914-950, 2020.

[10] D. Boffi, F. Brezzi, and M. Fortin. Mixed finite element methods and applications, volume 44 of Springer Series in Computational Mathematics. Springer, Heidelberg, 2013.

[11] D. Braess. Finite elements. Cambridge University Press, Cambridge, third edition, 2007. Theory, fast solvers, and applications in elasticity theory, Translated from the German by Larry L. Schumaker.

[12] A. Brandt. Multi-level adaptive solutions to boundary-value problems. Math. Comp., 31(138):333390, 1977.

[13] P. Destuynder and B. Métivet. Explicit error bounds in a conforming finite element method. Math. Comp., 68(228):1379-1396, 1999.

[14] A. Ern, I. Smears, and M. Vohralík. Discrete $p$-robust $\boldsymbol{H}$ (div)-liftings and a posteriori estimates for elliptic problems with $H^{-1}$ source terms. Calcolo, 54(3):1009-1025, 2017.

[15] A. Ern and M. Vohralík. Adaptive inexact Newton methods with a posteriori stopping criteria for nonlinear diffusion PDEs. SIAM J. Sci. Comput., 35(4):A1761-A1791, 2013.

[16] A. Ern and M. Vohralík. Stable broken $H^{1}$ and $\boldsymbol{H}$ (div) polynomial extensions for polynomialdegree-robust potential and flux reconstruction in three space dimensions. Math. Comp., 89(322):551-594, 2020.

[17] G. H. Golub and G. Meurant. Matrices, moments and quadrature with applications. Princeton Series in Applied Mathematics. Princeton University Press, Princeton, NJ, 2010. 
[18] W. Hackbusch. Multigrid methods and applications, volume 4 of Springer Series in Computational Mathematics. Springer-Verlag, Berlin, 1985.

[19] F. Hecht. New development in FreeFem++. J. Numer. Math., 20(3-4):251-265, 2012.

[20] F. Hecht, O. Pironneau, J. Morice, A. Le Hyaric, and K. Ohtsuka. FreeFem++. Technical report, Laboratoire Jacques-Louis Lions, Université Pierre et Marie Curie, Paris, http: //www . freefem. org/, 2012.

[21] B. Janssen and G. Kanschat. Adaptive multilevel methods with local smoothing for $H^{1}$ - and $H^{\text {curl }}$ conforming high order finite element methods. SIAM J. Sci. Comput., 33(4):2095-2114, 2011.

[22] P. Jiránek, Z. Strakoš, and M. Vohralík. A posteriori error estimates including algebraic error and stopping criteria for iterative solvers. SIAM J. Sci. Comput., 32(3):1567-1590, 2010.

[23] R. Luce and B. I. Wohlmuth. A local a posteriori error estimator based on equilibrated fluxes. SIAM J. Numer. Anal., 42(4):1394-1414, 2004.

[24] D. Meidner, R. Rannacher, and J. Vihharev. Goal-oriented error control of the iterative solution of finite element equations. J. Numer. Math., 17(2):143-172, 2009.

[25] G. Meurant and P. Tichý. Approximating the extreme Ritz values and upper bounds for the $A$-norm of the error in CG. Numer. Algorithms, 82(3):937-968, 2019.

[26] A. Miraçi, J. Papež, and M. Vohralík. A multilevel algebraic error estimator and the corresponding iterative solver with $p$-robust behavior. SIAM J. Numer. Anal., 58(5):2856-2884, 2020.

[27] P. Oswald. Multilevel finite element approximation. Teubner Skripten zur Numerik. [Teubner Scripts on Numerical Mathematics]. B. G. Teubner, Stuttgart, 1994. Theory and applications.

[28] J. Papež, U. Rüde, M. Vohralík, and B. Wohlmuth. Sharp algebraic and total a posteriori error bounds for $h$ and $p$ finite elements via a multilevel approach. Recovering mass balance in any situation. Comput. Methods Appl. Mech. Engrg., 371:113243, 2020.

[29] J. Papež, Z. Strakoš, and M. Vohralík. Estimating and localizing the algebraic and total numerical errors using flux reconstructions. Numer. Math., 138(3):681-721, 2018.

[30] U. Rüde. Fully adaptive multigrid methods. SIAM J. Numer. Anal., 30(1):230-248, 1993.

[31] U. Rüde. Mathematical and computational techniques for multilevel adaptive methods, volume 13 of Frontiers in Applied Mathematics. Society for Industrial and Applied Mathematics (SIAM), Philadelphia, PA, 1993.

[32] U. Rüde. Error estimates based on stable splittings. In Domain decomposition methods in scientific and engineering computing (University Park, PA, 1993), volume 180 of Contemp. Math., pages 111-118. Amer. Math. Soc., Providence, RI, 1994.

[33] Y. Saad. Iterative methods for sparse linear systems. Society for Industrial and Applied Mathematics, Philadelphia, PA, second edition, 2003.

[34] H. Si. TetGen, a Delaunay-based quality tetrahedral mesh generator. ACM Trans. Math. Software, 41(2):Art. 11, 36, 2015.

[35] Z. Strakoš and P. Tichý. On error estimation in the conjugate gradient method and why it works in finite precision computations. Electron. Trans. Numer. Anal., 13:56-80, 2002. 